\title{
Analysis of the Association Between the Incidence of Two Spatially Aggregated Foliar Diseases of Strawberry
}

\author{
W. W. Turechek and L. V. Madden
}

First author: Department of Botany and Plant Pathology, Oregon State University, 2082 Cordley Hall, Corvallis 97331-2902; and second author: Department of Plant Pathology, The Ohio State University, Ohio Agricultural Research and Development Center, Wooster 44691-4096. Accepted for publication 6 October 1999.

\begin{abstract}
Turechek, W. W., and Madden, L. V. 2000. Analysis of the association between the incidence of two spatially aggregated foliar diseases of strawberry. Phytopathology 90:157-170.

Association of the incidence of leaf blight (caused by Phomopsis obscurans) and leaf spot of strawberry (caused by Mycosphaerella fragariae) was assessed at multiple scales in perennial plantings at several commercial farms over 3 years (1996 to 1998). For each field, the presence or absence of each disease was recorded from $n=15$ leaflets in each of $N \approx 70$ evenly spaced sampling units, and the proportion of leaflets with blight, spot, and total disease (blight or spot) was determined. Individual diseases and total disease incidence were all well described by the beta-binomial distribution but not by the binomial distribution, indicating overdispersion of disease. The Jaccard similarity index was used to measure disease co-occurrence at the leaflet, sampling-unit, and field scales. Standard errors of this index for the lower two scales were obtained using the jackknife (resampling) procedure, and data randomizations were used to determine the expected Jaccard index for an independent arrangement of the two diseases, conditioned on the incidence and spatial heterogeneity of the

and cross-correlation statistics were calculated to determine the degree of covariation in incidence between the two diseases. Additionally, covariation between diseases was tested using a new procedure in the Spatial Analysis by Distance IndicEs (SADIE) class of tests. Covariation was detected in $21 \%$ of the data sets using rank correlation methods and in $15 \%$ of the data sets using the SADIE-based approach. The discrepancy between these two methods may be due to the rank correlation procedure not taking into account the effects of spatial pattern of disease incidence. There was no relationship between mean disease incidence per field of spot and blight or between degree of heterogeneity of the two diseases (as measured by $\theta$ of the beta-binomial distribution), demonstrating lack of covariation at the field scale. Incidence of leaflets with either disease (total disease incidence) could be well predicted using a linear combination of the estimated probabilities of leaf blight and leaf spot incidence based on independence of the two diseases. Heterogeneity of total disease incidence, measured with the estimated $\theta$ parameter of the beta-binomial distribution, could also be well predicted using a linear combination of the weighted $\hat{\theta}$ values for leaf blight and leaf spot, with weights proportional to incidence of the individual diseases.
\end{abstract} observed disease data. Results based on these statistics showed that only 4 of 52 data sets at the leaflet level and no data sets at the sampling-unit level had Jaccard index values significantly different from that expected under an independent rearrangement of the two diseases. Rank correlation
Additional keywords: aggregation, dispersion, quantitative epidemiology, statistical ecology.
The ecological association between pathogen species, and the diseases they cause, may have a significant influence on the spatiotemporal dynamics of disease epidemics and strongly influence which diseases dominate in a given habitat, in which habitat can refer to areas as large as several states or as small as single leaflets $(27,29)$. Two properties that help to characterize the nature of an association are occurrence and covariation (17). Occurrence (or co-occurrence) refers to the tendency for diseases to occupy the same habitat. When diseases are frequently found together, they are said to be associated; conversely, if diseases are infrequently found together, they are said to be dissociated. Covariation refers to the tendency of one disease intensity (incidence or severity) to increase or decrease in response to a change in intensity of another disease. If intensity of one disease increases in response to an increase in intensity of another disease, they are said to be positively correlated; similarly, if intensity of one disease decreases in response to an increase in intensity of another disease, they are said to be negatively correlated. Generally, one uses different tests for evaluating co-occurrence and covariation (17).

The influence that one disease has on another may be positive, negative, or neutral (17). For example, associations may result when

Corresponding author: L. V. Madden; E-mail address: madden.1@ osu.edu

Publication no. P-1999-1215-01R

(c) 2000 The American Phytopathological Society one pathogen wounds its host upon entry, thus producing favorable infection sites for another (e.g., potato early dying) $(26,46)$. Associations may also occur because of indirect effects, such as when both diseases are favored by the same physical environment. Dissociations may occur as a result of antibiotic production (antibiosis), competition (either direct or indirect), and systemically acquired resistance $(1,40,44,45)$. In many cases, more than one mechanism may determine the nature of an interaction $(44,45)$. Surprisingly, there have been very few studies dealing with the ecological association of plant diseases in commercial fields $(27,29,46)$.

The association among diseases is closely linked to the spatial pattern of the individual diseases. Perry (33) states "the definition of association is not easy, because it may not be possible to consider it in isolation from the spatial pattern of its two component species." Furthermore, the naturally occurring arrangement of diseased individuals could limit the range of possible associations or amplify the association found with a random disease pattern. Nonetheless, it is thought that if two diseases are closely associated, then their spatial patterns should be alike in some sense (27). This may not be the case for dissociated or independently distributed diseases, whose spatial patterns may or may not be alike.

Determining the scale of association (or lack of) is as important as determining the nature of an association (positive, negative, or neutral) and may provide substantial information on the epidemiology of the component species (27). For example, diseases associated at the field level (both diseases either present or not present 
in fields) may not be associated at the plant or leaf level (27). Associations of diseases at the field level may be the result of environmental (e.g., climatic) and biological conditions being met for both diseases over large scales (27), and dissociations at the leaf level in the same fields could be due to antibiosis or competition of the pathogens for infection sites.

There are many measures of pairwise associations (16), with several of these based on the presence or absence of the two species (diseases) in a collection of sampling units $(16,17)$. The Jaccard similarity index is one commonly used measure of co-occurrence (17), which is simply the proportion of sampling units where both diseases occur relative to the total number of sampling units where at least one of the species occur. However, the sampling distribution of the Jaccard index (and other similar indices) is unknown and, consequently, this index cannot be directly used to statistically test for species association without additional work.

Resampling methods provide a means to test the Jaccard index in a statistical hypothesis $(6)$. The $\chi^{2}$ test of independence $(16,17)$ can also be used for detecting associations, but this test can be misleading because it uses information on the sampling units where neither species occurs. It has been argued that the use of joint absences as a measure of species affinity may be inappropriate, thus limiting the usefulness of the $\chi^{2}$ test $(16,17)$. Moreover, the $\chi^{2}$ test, as well as standard tests based on the Jaccard and similar indices $(16,17)$, assumes that the individual species are distributed randomly. This assumption is not likely to hold for many plant diseases, since most are aggregated in fields $(19,21,22,42,43)$. Although various methods have been proposed for correcting the $\chi^{2}$ statistic for aggregation $(2,4,9,36)$, the use of joint absences can still limit the $\chi^{2}$ test. Using resampling techniques and a randomization test, we will show that the Jaccard similarity index can be used to test the null hypothesis of independence of two diseases while conditioning results on the spatial information in the data.

Correlation coefficients $\left(\rho_{0}\right)$ are standard statistics used to quantify species covariation (17) at the level of the sampling unit. Crosscorrelation coefficients can be used to characterize covariation that is not aligned exactly with the sampling units, such as when a high intensity of disease one is associated with a high intensity of disease two in neighboring sampling units, but not necessarily in the same sampling units. However, correlation coefficients do not explicitly account for the spatial pattern of the individual diseases. Recently, Perry extended the novel Spatial Analysis by Distance IndicEs (SADIE) system of pattern analysis $(32,33)$ to include a test for species covariation that is specifically designed for use with count data, conditions results on the spatial pattern of both species, and considers the influence of species from neighboring sampling units $(33,35)$.

Strawberry leaf blight (caused by Phomopsis obscurans) and strawberry leaf spot (caused by Mycosphaerella fragariae [anamorph: Ramularia brunnea]) are two common foliar diseases of Ohio-grown strawberries (Fragaria $\times$ ananassa) $(7,8)$. Severe epidemics of either of these two diseases can lead to reduction in the photosynthetic capacity of the plant. In Ohio, where strawberries are grown as a perennial crop, this may damage the winter hardiness of the crop, which may significantly affect yield. Extensive spatial pattern analyses have been published for both of these diseases $(42,43)$. However, little is known about the association of these two diseases in strawberry fields. A fuller understanding of the interaction between these two pathogens will provide a better understanding of their joint epidemiology $(28,29)$.

When leaf blight and leaf spot both are present in a field, it may be of interest to determine the overall incidence of leaf disease (proportion of leaflets with either blight or spot) rather than the incidence of each disease separately (28). From a practical standpoint, this information is easier to collect in surveys, especially by lessexperienced scouts, and provides useful information for disease management since both diseases are controlled the same way $(7,8)$. Furthermore, some mechanistic models of crop loss are driven by the disease-free leaf area, not by the individual disease intensities (24). Thus, it is beneficial to determine how incidence and heterogeneity of component diseases, and their associations (if any), affect the incidence and heterogeneity of total or combined disease incidence.

The objectives of this research were to (i) determine the ecological associations between Phomopsis leaf blight and strawberry leaf spot disease in commercial perennial strawberry fields where both diseases occur; (ii) determine the similarity of incidence and heterogeneity of the two diseases in fields where both diseases occur; (iii) determine the effect of hierarchical scale on associations of disease; (iv) introduce a randomization procedure to test the significance of the Jaccard similarity index for diseases that are spatially aggregated; and (v) determine the effects of incidence and heterogeneity of leaf spot and blight on total (combined) disease.

\section{MATERIALS AND METHODS}

Data collection. Naturally occurring epidemics of Phomopsis leaf blight and strawberry leaf spot were monitored over 3 years. In 1996 and 1997, the diseases were assessed at a single commercial strawberry farm in Ohio. This was one of the two farms sampled by Turechek and Madden (42). The farm was located approximately $22 \mathrm{~km}$ southwest of Columbus in Darbydale, $\mathrm{OH}\left(39^{\circ} 51^{\prime} 13^{\prime \prime} \mathrm{N}\right.$, $83^{\circ} 10^{\prime} 59^{\prime \prime} \mathrm{W}$ ), and had several perennial plantings (fields) comprising 10 to 20 rows of strawberry each. The plantings ranged in age from 1 to 5 years. Plants were grown in a matted row system, approximately $0.3 \mathrm{~m}$ wide grown on 1 -m centers. 'Earliglow' and 'Honeoye' were the dominant cultivars grown. Other cultivars rated for disease included 'Delmarvel' and 'Primetime'.

In 1996, sampling started on 13 July and continued until 13 September. In 1997, sampling started on 29 April and continued until 26 September. Sampling occurred at approximately biweekly intervals in both years. These dates encompassed time of fruit development, harvesting (fruit maturity), renovation (mowing of leaves after harvest), and regrowth of vegetation. Three or four fields were randomly selected at each sampling date (independent of disease status) for disease assessment from a total of eight or nine fields over these 2 years. Not all fields were sampled the same number of times. The same leaves were not necessarily sampled in different assessments of the same field.

In 1998, a statewide survey of foliar diseases of strawberry was conducted on commercial farms from 16 June through 23 June. This time was chosen to coincide with harvesting. Farms selected for the survey were chosen in a stratified random sample. In this survey, the state was divided into nine regions or strata, and commercial strawberry farms within these regions were identified from the member directories of the National Association of Strawberry Growers and the Ohio Small Fruit Growers Association. From the compiled listing, three or four farms were randomly chosen from each of the nine regions or strata (except in two of the southern regions, in which only one and two strawberry farms could be identified). A total of 26 farms was surveyed in 1998 (this did not include the farm assessed in 1996 and 1997, although it was included in the farm listing for potential selection) and, for each farm, three fields were randomly chosen from the fields identified for disease assessment.

Total area devoted to strawberry production on these 26 farms ranged from approximately 14 ha to less than 1 ha. The perennial plantings ranged in age from 1 to 7 years. Cultivars assessed for disease included 'Earliglow', 'Honeoye', 'Jewel', 'Allstar', 'Red Chief', 'Sure Crop', 'Cavendish', and 'Noreaster'. All cultivars are day-length sensitive (June-bearers) (42,43).

Fields were sampled almost identically over the 3 years. Details are given by Turechek and Madden (43), and a summary of the method is presented here. For each field and assessment time, a single row was randomly chosen as the primary transect. In 1996 and 1997, a short secondary transect was arbitrarily chosen to ap- 
proximately bisect the field; this transect was not included in the 1998 samplings. The initial sampling unit was arbitrarily chosen within the first 20 "steps" of the primary transect and each subsequent sampling unit was located at a distance of three steps $(\sim 2.5 \mathrm{~m})$ from the previous sampling unit, assuring approximately evenly spaced sampling units. In 1996 and 1997, a sampling unit in each row also was sampled along the secondary (bisecting) transect. The number of sampling units, $N$, varied from 27 to 87 depending upon the field size, but was typically between 65 and 75 . Sampling units consisted of 15 leaflets (five leaves of three leaflets each). Each leaflet was rated for the presence or absence of leaf blight and leaf spot symptoms. This is a form of cluster sampling (14) in which the clusters (sampling units) were chosen systematically and all individuals (e.g., leaflets) per sampling unit $(n)$ were assessed.

In 1996 and 1997, 46 data sets were collected in total. Twentythree of these had leaflets with both leaf blight and leaf spot symptoms. In 1998, 16 out of the 26 farms surveyed had fields that had leaflets with both blight and spot, primarily in the southern half of the state. From the possible 48 fields ( 16 farms $\times 3$ fields each), 29 had both diseases. In total, 124 data sets were collected from 1996 to 1998, and 52 of these (the ones with both leaf blight and leaf spot) were used in the analyses presented below. Twenty-three of these fifty-two data sets were used by Turechek and Madden (42) and all 52 were used by Turechek and Madden (43) for describing the spatial patterns of leaf blight and leaf spot, respectively. In the previous papers, there was no consideration of total disease incidence nor was there any direct comparison of the properties of the two diseases within specific fields or sampling units.

Disease assessment and pattern analysis. Distribution analysis. For each of the 52 individual field assessments, the beta-binomial $(13,21)$ and the binomial distributions were fitted to the data using BBD (20) for each disease separately and then for total disease (a leaflet was considered diseased if it had either leaf blight or leaf spot symptoms). The binomial distribution has a single parameter representing the probability of disease $(\pi)$. The beta-binomial has two parameters, $p_{t}$, which is the expected probability of a diseased leaflet for the field, and $\theta$, which is a measure of the variation or heterogeneity in disease incidence per sampling unit for a given field. A good fit to the binomial distribution is suggestive of a random spatial pattern of disease incidence, while a good fit to the beta-binomial distribution is suggestive of an aggregated pattern of disease incidence (21). A log-likelihood ratio test statistic (LRS) was calculated for each data set to determine if the beta-binomial fitted the data better than did the binomial (47).

Symbol and subscript notation. A note on terminology is needed. Where applicable, a term followed by only the " $t$ " or " $s u$ " subscript refers to disease information (leaf blight, leaf spot, or total disease) at the leaflet and sampling- $u$ nit levels, respectively; and a term without the " $t$ " or "su" subscripts refers to the term in general. Also, if a term is only relevant at the leaflet level (e.g., $\theta$ ), then the " $t$ " subscript is not used.

Terms followed by the " $i$ " subscript refer to leaflet characteristics or measurements in the $i$ th $(i=1,2, \ldots, N)$ sampling unit. The statistic $a_{i}$ refers to the number of leaflets ( 0 to $n$ ) with both blight and spot symptoms within the $i$ th sampling unit; $b_{i}$ refers to the number of leaflets with only blight symptoms within the $i$ th sampling unit; $c_{i}$ refers to the number of leaflets with only spot symptoms within the $i$ th sampling unit; and $d_{i}$ refers to the number of diseasefree leaflets within the $i$ th sampling unit. Let $x_{i}=a_{i}+b_{i}$ refer to the number of leaflets with blight within a sampling unit, $y_{i}=a_{i}+$ $c_{i}$ refer to the number of leaflets with spot within a sampling unit, and $z_{i}=a_{i}+b_{i}+c_{i}$ refer to the number of leaflets within a sampling unit with either blight or spot symptoms (total diseased leaflets).

Let $a_{t}=\Sigma a_{i}, b_{t}=\Sigma b_{i}, c_{t}=\Sigma c_{i}$, and $d_{t}=\Sigma d_{i}$ be the number of leaflets with blight and spot on the same leaflets, the number of leaflets with blight only, the number of leaflets with spot only, and the number of disease-free leaflets, respectively, for an entire field (data set) of $n N$ leaflets. Therefore, $x_{t}=a_{t}+b_{t}$ refers to the number of leaflets with blight in a field; $y_{t}=a_{t}+c_{t}$ refers to the number of leaflets with spot in a field; and $z_{t}=a_{t}+b_{t}+c_{t}$ refers to the number of leaflets with either blight or spot in a field. Moreover, let $\hat{p}_{t, x}=$ $\left(a_{t}+b_{t}\right) /\left(a_{t}+b_{t}+c_{t}+d_{t}\right)$ be the moment estimate of the probability that a leaflet has leaf blight symptoms, $\hat{p}_{t, y}=\left(a_{t}+c_{t}\right) /\left(a_{t}+b_{t}+c_{t}+d_{t}\right)$ be the moment estimate of the probability that a leaflet has leaf spot symptoms, and $\hat{p}_{t, z}=\left(a_{t}+b_{t}+c_{t}\right) /\left(a_{t}+b_{t}+c_{t}+d_{t}\right)$ be the moment estimate of the probability that a leaflet has either leaf blight or leaf spot symptoms (total disease incidence). In general, $x, y$, and $z$ are used as subscripts to identify terms dealing with specific diseases or total disease.

Terms followed by the " $i, s u$ " subscript refer to sampling-unitscale characteristics in the $i$ th sampling unit. At the sampling-unit level, $a_{i, s u}, b_{i, s u}, c_{i, s u}, d_{i, s u}, a_{s u}$ (e.g., $\left.a_{s u}=\Sigma a_{i, s u}\right), b_{s u}, c_{s u}, d_{s u}, x_{s u}, y_{s u}$, $z_{s u}, p_{s u, x}, p_{s u, y}$, and $p_{s u, z}$ then replace $a_{i}, b_{i}, c_{i}, d_{i}, a_{t}, b_{t}, c_{t}, d_{t}, x_{t}, y_{t}, z_{t}$, $p_{t, x}, p_{t, y}$, and $p_{t, z}$, respectively. Measurements are made over the $N$ sampling units rather than the $n N$ leaflets. The terms $a_{i, s u}, b_{i, s u}, c_{i, s u}$, and $d_{i, s u}$ represent mutually exclusive binary events ( 0 or 1$)$; that is, one of the four statistics must equal 1 , while the others must equal 0 for the classification of a sampling unit.

Binary power law analysis. The binary form of Taylor's power law was used to assess the degree of heterogeneity across data sets. The binary power law can be expressed as a relationship between two variances, the observed sample variance of diseased leaflets $\left(v_{(o b s)}\right)$ and the theoretical variance of a random distribution $\left(v_{(r a n)}\right)(12,23)$. With binary or proportion data, $v_{(\text {ran })}$ is the binomial variance, $n p_{t}\left(1-p_{t}\right)$, in which $n=15$ (leaflets per sampling unit) here. Linearization of the power law relationship is accomplished via logarithmic transformation to give $\ln \left(v_{(o b s)}\right)=$ $\ln (A)+\beta \ln \left[n p_{t}\left(1-p_{t}\right)\right]$, in which $\ln (A)$ and $\beta$ are the intercept and slope, respectively, of a straight line (43).

Parameters of the binary power law were estimated by regression for each disease separately and then for total disease. In fitting the power law to the data, we used estimated $p_{t}$ from the betabinomial distribution $\left(\hat{p}_{t, x}, \hat{p}_{t, y}\right.$, or $\left.\hat{p}_{t, z}\right)$ and the moment estimate of $v_{(o b s)}\left(v_{(o b s), x}, v_{(o b s), y}\right.$, and $\left.v_{(o b s), z}\right)$ for each data set. When $A$ and $\beta$ are both equal to 1 , a random pattern of disease as described by the binomial distribution is indicated. When $\beta=1$ and $A>1$, there is overdispersion, but the degree of overdispersion for each data set (e.g., $\theta$ of the beta-binomial) does not depend on $p_{t}$. When $\beta$ and $A$ are greater than 1 , the degree of overdispersion or heterogeneity changes with $p_{t}(23)$.

Autocorrelation analysis. First-order spatial autocorrelation coefficients were calculated for each of the 52 individual data sets for each disease separately and then for total disease. First-order autocorrelation coefficients $\left(r_{1}\right)$, based on logits of disease incidence $(w)$, were calculated using

$$
\hat{r}_{1}=\frac{\sum_{i=1}^{N-1}\left(w_{i}-\bar{w}\right)\left(w_{i+1}-\bar{w}\right)}{\sum_{i=1}^{N}\left(w_{i}-\bar{w}\right)^{2}}
$$

in which $w_{i}=\ln \left[l_{i}^{\prime} /\left(1-l_{i}^{\prime}\right)\right], \bar{w}=\Sigma w_{i} / N, l_{i}^{\prime}=\left(l_{i}+0.5\right) /(n+1)$, and $l_{i}$ is the number of diseased leaflets in the $i$ th sampling unit $\left(x_{i}, y_{i}\right.$, or $\left.z_{i}\right)$. The corrected value of disease proportion $\left(l_{i}^{\prime}\right)$, known as the Haldane correction, was used to avoid taking logarithms of 0 (42). A data set was considered to exhibit significant autocorrelation if the coefficient exceeded the bounds of its approximate 95\% confidence interval (calculated as $1.645 / \sqrt{ } N$ ).

SADIE. The SADIE program was used to calculate the distance to regularity $\left(D_{\mathrm{r}}\right)(32,34)$ using the data from the primary transect for each of the 52 individual field assessments for each disease separately and then for total disease. Details were given by Turechek and Madden $(42,43)$. In brief, to calculate $D_{\mathrm{r}}$, SADIE used an algorithm that produced a regular arrangement of counts (in which each sampling unit contains $x_{t} / N, y_{t} / N$, or $z_{t} / N$ diseased individ- 
uals) from a transect (or map) of spatially referenced counts by moving individuals (diseased leaflets here) from one location (sampling unit) to another via the shortest possible route (distance). Summing the distances that each individual moved in order to achieve regularity gave the distance to regularity. A randomization test was used to determine if the observed distance to regularity was a particularly large or small value. To perform this test, counts for each sampling unit $\left(x_{i}, y_{i}\right.$, or $\left.z_{i}\right)$ were randomly rearranged among the $N$ sampling units and, from this new random arrangement, a new distance to regularity was calculated. For our analyses, 100 randomizations were performed on each data set and, from these randomizations, a frequency distribution of distances was derived. Percentiles were calculated from this frequency distribution, and the significance level for a one-sided test for aggregation was determined from the proportion of randomizations with distances to regularity as great or greater than $D_{\mathrm{r}}$.

An index of aggregation $\left(I_{\mathrm{a}}\right)$ based on the SADIE methodology can be written as $I_{\mathrm{a}}=D_{\mathrm{r}} / E_{\mathrm{a}}$, in which $E_{\mathrm{a}}$ is the average (expected) distance to regularity for the 100 randomization results $(32,34)$. A value of $I_{\mathrm{a}}=1$ suggests a spatially random pattern; $I_{\mathrm{a}}>1$ suggests a more aggregated pattern; and $I_{\mathrm{a}}<1$ suggests a more regular pattern.

Jaccard association analysis. The Jaccard similarity index $(J)$ was used as a measure of co-occurrence between blight and spot $(16,17)$ at the leaflet and higher scales. For each data set, the index was calculated using $J=a /(a+b+c)$ or, more specifically, $J_{t}=a_{t}$ $/\left(a_{t}+b_{t}+c_{t}\right)$ and $J_{s u}=a_{s u} /\left(a_{s u}+b_{s u}+c_{s u}\right)$ for the leaflet and sampling-unit scales, respectively. The number of leaflets (or sampling units) with neither disease $\left(d_{t}\right.$ or $\left.d_{s u}\right)$ is not used to calculate $J$. The index represents the proportion of leaflets (or sampling units) that have both diseases relative to the number of leaflets (or sampling units) that have either disease. Values of the index lie between 0 and 1 . Relatively large values of the index suggest a positive association between diseases (and by inference, pathogens), while relatively small values suggest a negative association or dissociation between diseases. Standard use of the Jaccard index assumes that populations are distributed randomly in space. Thus, the unmodified value of the index may not provide a good measure of association when considerable heterogeneity (overdispersion) exists for one or both of the diseases (16).

Because the sampling distribution of the Jaccard index is unknown, standard errors of the Jaccard index are not directly estimable (6). However, a resampling estimate of the standard error can be obtained using the jackknife procedure (6). For each data set, the jackknifed standard error was calculated as follows: (i) calculate the (standard) Jaccard coefficient $(J)$ as described above; (ii) remove the first observation and recalculate the coefficient based on the remaining data points $\left(J_{-1}\right)$; (iii) repeat step ii for each observation in turn (e.g., to obtain $J_{-i}$ for the $i$ th out of $N$ observations) in order to calculate $N$ different $J_{-i}$ values; and (iv) calculate the $i$ th pseudovalue $\left(v_{i}\right)$ for each observation as $v_{i}=J+(N-1)\left(J-J_{-i}\right)$. The jackknifed standard error of the Jaccard coefficient then is

$$
s_{J}=\sqrt{\frac{\sum\left(v_{i}-\bar{v}\right)^{2}}{N(N-1)}}
$$

Jackknife standard errors were calculated using this algorithm for each data set at both the leaflet and sampling-unit scales. At the leaflet level, the cluster sampling was preserved in that the $i$ th observation corresponded to all 15 leaflets, in the $i$ th sampling unit; thus, each $-i$ was obtained by removing the entire cluster of 15 leaflets. This corrected for the overdispersion or heterogeneity of the data by preserving the spatial information contained within clusters (sampling units). At the sampling-unit level, the $i$ th observation corresponds to a single number ( 0 or 1 ) for each disease.

Randomizations. It is not clear for a set of data what value of the Jaccard index constitutes independence between the two diseases; that is, the expected value of $J$ under the null hypothesis of independence is not known. To resolve this problem, data ran- domizations were used to calculate an average value of the index

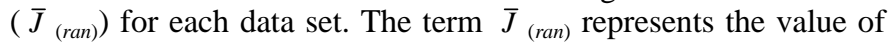
the Jaccard similarity index one would expect if the two diseases were distributed independently, given the observed incidence (e.g., $\hat{p})$ and spatial structure $\left(\theta, I_{\mathrm{a}}\right)$ for the two diseases. $\bar{J}_{(\text {ran })}$ was calculated for each data set at both the leaflet scale $\left(\bar{J}_{t, \text { (ran })}\right)$ and the sampling-unit scale $\left(\bar{J}_{s u,(r a n)}\right)$ using the observed data. Randomization methods are presented in the Appendix.

To test whether the observed Jaccard similarity index was significantly different from its corresponding $\bar{J}_{t,(\text { ran })}\left(\right.$ or $\left.\bar{J}_{s u,(r a n)}\right)$, we assumed a normal distribution for the index with the mean estimated by $J$ ( $J_{t}$ or $J_{s u}$, determined directly from the data) and the standard error estimated with the jackknifed value, $s_{J}(25)$. The following standard normal test statistic was calculated.

$$
Z=\frac{J-\bar{J}_{(\text {ran })}}{s_{J}}
$$

The leaflet and sampling-unit Jaccard values were substituted for $J$ and $\bar{J}_{(\text {ran })}$ in equation 3 . Treating equation 3 as a two-sided test, values of $Z>1.96$ indicate significant positive association and $Z<$ -1.96 suggest negative associations at $P=0.05$. This test was performed on each data set at both the leaflet and sampling-unit scale.

Extreme Jaccard values. Maximum and minimum values of the Jaccard index were calculated at both the leaflet and sampling-unit levels to derive the range of possible Jaccard values $\left(J_{t,(\max )}, J_{t,(\min )}\right.$, $J_{s u,(\max )}$, and $\left.J_{s u,(\min )}\right)$ for each data set (presented in the Appendix). As above, these Jaccard values were based on the constraint that incidence of the individual diseases at both levels and heterogeneity at the leaflet level be the same as the observed values. This preserved the spatial attributes of the data and conditioned association results on the distributions of the two individual diseases.

Covariation analyses. Covariation between the two diseases was determined using Spearman's rank correlation statistic (17). Rank correlations provide a nonparametric measure of the correlation of disease incidence among the sampling units. For each data set, the vectors $\boldsymbol{X}_{\boldsymbol{t}}=\left[x_{1}, x_{2}, \ldots, x_{N}\right]^{\prime}$ and $\boldsymbol{Y}_{\boldsymbol{t}}=\left[y_{1}, y_{2}, \ldots, y_{N}\right]^{\prime}$ were created, in which $x_{i}$ and $y_{i}$ were defined previously and the prime symbol denotes the matrix transpose operator. The elements of each of the vectors were separately ranked to obtain $\boldsymbol{Q}_{t}$ and $\boldsymbol{U}_{t}$ for $\boldsymbol{X}_{t}$ and $\boldsymbol{Y}_{t}$, respectively (ties were handled using the average value of their ranks). For instance, $q_{1}$ is the rank of $x_{1}$ in the $\boldsymbol{X}_{\boldsymbol{t}}$ vector and $u_{N}$ is the rank of $y_{N}$ in the $\boldsymbol{Y}_{\boldsymbol{t}}$ vector. Spearman's rank correlation was calculated using

$$
\hat{\rho}_{k}=\frac{\sum_{i=1}^{N-k}\left(q_{i+k}-\bar{q}\right)\left(u_{i}-\bar{u}\right)}{\left[\sum_{i=1}^{N-k}\left(q_{i+k}-\bar{q}\right)^{2} \sum_{i=1}^{N}\left(u_{i}-\bar{u}\right)^{2}\right]^{1 / 2}}
$$

in which $\bar{q}$ and $\bar{u}$ are the averages of the $N q_{i}$ and $u_{i}$ ranks, and $k=$ $0 ; k$ is an index for the spatial separation of the two variables $(q$ and $u$ ). In nominal calculations, $k=0$, indicating that incidence values in the same sampling units are being compared. If species are associated, the large ranks of each variable will occur together, thus making $\hat{\rho}_{0}$ large and positive. Conversely, if species are dissociated, the large ranks of one variable will occur with the smaller ranks of another variable, giving large negative $\hat{\rho}_{0}$ values.

Equation 4 was also used to calculate the $k$ th cross-correlation coefficient (for $k \neq 0$ ). Cross-correlation coefficients measure the correlation between species $k$ sampling units away. For example, $\rho_{2}$ is the measure of the correlation between blight incidence and spot incidence two sampling units apart. For each data set, crosscorrelation coefficients were calculated from $k=-5$ to $k=5$ using Minitab (version 12; Minitab Inc., State College, PA).

A measure of covariation using the SADIE methodology. Perry $(33,35)$ has extended the use of the SADIE methodology to assess species covariation. The procedure tests the hypothesis that the diseases are dispersed at random with respect to each other. 
The method detects similar properties of association as $\rho_{k}$, but explicitly conditions results on the spatial patterns of each disease, and is also based on properties of discrete data such as counts.

The following is a paraphrasing of Perry's (33) original description of the procedure. Consider the vectors $\boldsymbol{X}_{\boldsymbol{t}}$ and $\boldsymbol{Y}_{\boldsymbol{t}}$ of dimension $N \times 1$ consisting of the number of leaflets with blight and spot, respectively, and let the vector $S_{t}=X_{t}+Y_{t}$ represent the sum of the blighted and spotted leaflets within sampling units; $s_{i}=x_{i}+y_{i}$, for the $i$ th sampling unit. (It should be noted that $s_{i}$ is not the same as $z_{i}$. The term $z_{i}$ is total disease incidence in the sampling unit, which cannot exceed $n ; s_{i}$ can range from 0 to $2 n$.) The counts in both vectors are scaled to compensate for differences in incidence of the two diseases, as described by Perry (35). If the diseases are highly associated, then the counts in $S_{t}$ will reinforce each other in their totals, in that associations of high or low counts of the two diseases will produce totals that are, relatively speaking, very high or very low. The range of $S_{t}$ values will then vary widely and any aggregation in the individual species will be enhanced in their totals. By contrast, dissociated counts compensate each other to produce a much more even set of total counts $\left(\boldsymbol{S}_{t}\right)$ covering a narrow range. Thus, any aggregation in the individual species will be masked in their totals. These features may be quantified by treating the totals $\left(\boldsymbol{S}_{t}\right)$ as a single species (variable) count and analyzing their distance to regularity using the standard SADIE method.

The distance to regularity of the observed totals $\left(\boldsymbol{S}_{t}\right.$; with vector elements $\left.s_{i}, i=1,2, \ldots, N\right), T$, is computed and stored as done for a single disease $(42,43)$. Random permutations (arrangements) of the counts for disease $x$ are then found in a separate step, which has very similar values of $D_{\mathrm{r}}$ (the observed distance to regularity of disease $x$ ) and $\delta$ (a small constant that is used to control 'edge effects') (33). For each of $j$ random permutations, the total is formed of this set of counts of disease $x$ and of the original counts of disease $y$, as above, and distance to regularity is determined. This value is called $T_{j(y) x}$. A randomization test is performed, as described for the single species case, from the observed $T$ (for the actual data) and the frequency distribution of the values of $T_{j(y) x}$. Furthermore, an index of association, $I_{(y) x}$, may be formed from $T / E_{(y) x}$, in which $E_{(y) x}$ represents the average value of $T_{j(y) x}$ over the randomizations. Values of $I_{(y) x}>1$ indicate positive association, $I_{(y) x}<1$ indicate negative association, and values close to 1 indicate a random placement of one disease with regard to the other. The entire procedure is then repeated, with diseases $y$ and $x$ reversed, for the observed value of $T$ and the randomized $T_{j(x) y}$ values. Perry (33) states that, in most cases, the test and index give very similar results for the two sets of randomizations, $(y) x$ and $(x) y$, so that they may then be combined to give an average probability, $P$, and index, $I$.

Field-level associations. Bivariate plots of $\hat{p}_{t, x}$ and $\hat{p}_{t, y}$ and of $\hat{\theta}_{x}$ and $\hat{\theta}_{y}$ (estimated incidence and heterogeneity of the two diseases) were used to determine if there was a quantitative field-level relationship in incidence and heterogeneity, respectively, between the two diseases. Rank correlation coefficients were calculated to determine the magnitude of the relationship, if any. Furthermore, from the total of 124 data sets collected over the 3 years (which included the 52 data sets with both diseases), the Jaccard index was calculated at the field level to determine if there was any association of the diseases in terms of their co-occurrences in entire fields.

Total disease incidence (blight or spot) was assessed in relation to the individual diseases. If blight and spot occur independently of each other, then the probability that a leaflet will have both leaf blight and leaf spot symptoms is $p_{t, x y}=p_{t, x} \cdot p_{t, y}$. From elementary probability theory, the probability $(\operatorname{Pr}[\cdot])$ that the leaflet will have any disease is then $\operatorname{Pr}\left[p_{t, x} \cup p_{t, y}\right]=p_{t, z, \text { (ind })}=p_{t, x}+p_{t, y}-p_{t, x} \cdot p_{t, y}$ (5), in which $p_{t, z, \text { ind })}$ is total disease incidence under the independence assumption. In terms of their estimates, $\hat{p}_{t, z, \text { (ind })}=\hat{p}_{t, x}+\hat{p}_{t, y}-$ $\hat{p}_{t, x} \cdot \hat{p}_{t, y}$. A regression of observed $\hat{p}_{t, z}$ on $\hat{p}_{t, z,(\text { ind })}$ will yield a slope of 1 and intercept of 0 if the two diseases occur independently of each other. Deviation from this prediction is evidence that the diseases are not independent at the field level. If blight and spot are (positively) associated, then the regression will yield a slope less than 1 and, similarly, if the diseases are dissociated (negatively associated), a slope greater than 1 .

Regressions were also performed by replacing observed $\hat{p}_{t, z}$ with their maximum and minimum values $\left(\hat{p}_{t, z,(\max )}\right.$ and $\left.\hat{p}_{t, z,(\min )}\right)$, previously determined based on rearrangements of diseased leaflets within sampling units (presented in the Appendix). These regressions were conducted to provide the range of possible slope and intercept values for the most extreme cases of $\hat{p}_{t, z}$.

An approximate estimate of heterogeneity of total disease incidence, based on the assumption that leaf blight and leaf spot are distributed independently of each other, was made using the formula for the moment estimate of $\theta$ (21). The prediction is given by

$$
\hat{\theta}_{z, \text { (ind })}=\left[v_{z}-n \hat{p}_{t, z, \text { ind })}\left(1-\hat{p}_{t, z, \text { ind })}\right)\right] /\left[n^{2} \hat{p}_{t, z, \text { ind })}\left(1-\hat{p}_{t, z, \text { ind })}\right)-v_{z}\right]
$$

in which

$$
v_{z}=n \hat{p}_{t, z, \text { ind })}\left(1-\hat{p}_{t, z, \text { ind })}\right)\left[1+\phi_{(w t d)}(n-1)\right]
$$

is the beta-binomial variance for $z_{(\text {ind })}\left(=n \hat{p}_{t, z,(i n d)}\right)$ and $\phi_{(w t d)}$ is a weighted estimate of the intracluster correlation coefficient (in general, $\phi=\theta /[1+\theta])$. The weighted estimate under independence is given by

$$
\phi_{(w t d)}=\frac{\hat{p}_{t, x}}{\hat{p}_{t, x}+\hat{p}_{t, y}} \hat{\phi}_{x}+\frac{\hat{p}_{t, y}}{\hat{p}_{t, x}+\hat{p}_{t, y}} \hat{\phi}_{y}
$$

in which $\hat{\phi}_{x}=\hat{\theta}_{x} /\left(1+\hat{\theta}_{x}\right)$ and $\hat{\phi}_{y}=\hat{\theta}_{y} /\left(1+\hat{\theta}_{y}\right)$ are the observed intracluster correlations for leaf blight and leaf spot, respectively (21). The contribution of each individual disease to the heterogeneity of total disease incidence in equation 7 is proportional to the incidence of each disease relative to total disease. For the data sets in which leaf blight and leaf spot exhibited significant covariation (discussed below), the covariance term $\left(\operatorname{Cov}\left[\hat{p}_{t, x}, \hat{p}_{t, y}\right]\right)$ was added to the right-hand side of equation 6 to determine $v_{z}$.

\section{RESULTS}

Incidence and heterogeneity of disease. Distribution analysis. The program BBD successfully calculated maximum likelihood estimates of $p_{t}$ and $\theta$ for 50,45 , and 51 of the 52 data sets for blight, spot, and total disease, respectively. A LRS showed that the frequency distribution of diseased leaflets could be described better by the beta-binomial rather than the binomial distribution in 51 of the 52 data sets for both blight and spot and in all data sets for total disease. Transect maps for six example fields in Figure 1 show the range of conditions encountered in this study.

Disease incidence and heterogeneity. Table 1 shows the minimum, first quartile, median (second quartile), third quartile, and maximum values for incidence at the leaflet level $\left(\hat{p}_{t}\right)$ and the sampling-unit level $\left(\hat{p}_{s u}\right)$ for the 52 data sets. The range of spot incidence values per field $\left(\hat{p}_{t, y}\right.$ and $\left.\hat{p}_{s u, y}\right)$ was larger than that of blight incidence values ( $\hat{p}_{t, x}$ and $\hat{p}_{s u, x}$ ); however, the distribution of $\hat{p}_{t, y}$ and $\hat{p}_{s u, y}$ values was much more skewed than that of $\hat{p}_{t, x}$ and $\hat{p}_{s u, x}$ values, as indicated by smaller values of the median and other quartiles for spot. For instance, $50 \%$ of the fields had blight incidence greater than 0.15 , whereas $50 \%$ had spot incidence greater than only 0.055 (Table 1). For these statistics, total disease incidence values $\left(\hat{p}_{t, z}\right.$ and $\left.\hat{p}_{s u, z}\right)$ must be greater than or equal to the maximum of $\hat{p}_{t, x}$ and $\hat{p}_{t, y}$, by definition.

The range of disease heterogeneity values for leaf spot $\left(\hat{\theta}_{y}\right)$ was much larger than that of heterogeneity values for either blight or total disease $\left(\hat{\theta}_{x}\right.$ or $\hat{\theta}_{z}$ ), and the largest values were found for leaf spot (Table 1). When the test could be performed, 49 out of 50,39 out of 45 , and 51 out of 51 of the $\hat{\theta}_{x}, \hat{\theta}_{y}$, and $\hat{\theta}_{z}$ values were significantly greater than 0 , according to a $t$ test, for the blight, 
spot, and total disease data sets, respectively, indicating substantial overdispersion (21). Of the seven values of $\hat{\theta}$ that were not significantly different from 0 , mean incidence $\left(\hat{p}_{t}\right)$ per field was less than 0.038 in these data sets.

Two of the example fields in Figure 1 (A and $\mathrm{C}$ for blight; and $\mathrm{B}$ and $\mathrm{C}$ for spot) had $\hat{\theta}$ values at or below the medians for all fields, and the other fields had larger $\hat{\theta}$ values (Table 2). Fields D and $F$ were characterized by large $\theta$ values for both diseases. A large $\theta$ is characteristic of high variation in the number of diseased leaflets per sampling unit.

Binary power law. The binary power law provided an excellent description of the relationship between the observed variance $\left(v_{(o b s)}\right)$ and the variance of the binomial distribution $\left(v_{(\text {ran })}\right)$ on a log scale for leaf blight, leaf spot, and total disease for the 3 years of data (Table 3). Estimated intercepts $(\ln [A])$ were significantly greater than 0 and slopes were significantly greater than 1 for leaf blight,

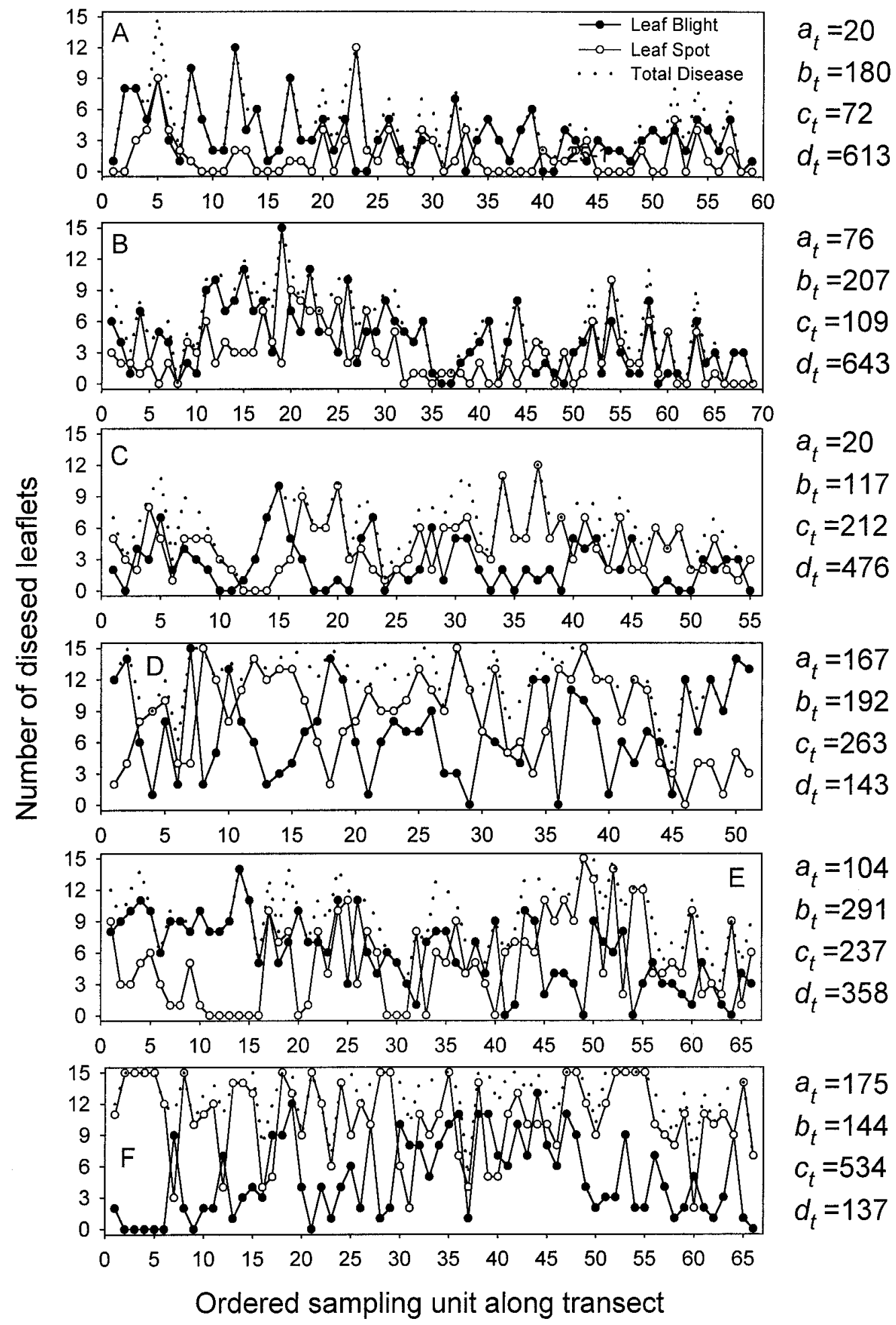

Fig. 1. Map of the number of diseased leaflets with Phomopsis leaf blight (solid circles), leaf spot (open circles), and total disease (blight or spot; dotted line) in individual sampling units ( $n=15$ leaflets) along a transect (with $N$ sampling units) for six example strawberry fields. For the entire field of $n N$ leaflets, $a_{t}$ is the number of leaflets with both diseases, $b_{t}$ is the number of leaflets with blight only, $c_{t}$ is the number of leaflets with spot only, and $d_{t}$ is the number of disease-free leaflets. Table 2 presents the statistics for the six fields. 
leaf spot, and total disease $(P<0.01)$. This indicated significant overdispersion of disease incidence across all data sets, and because $\hat{\beta}$ was greater than 1 , the degree of aggregation varied systematically with incidence for the individual diseases and total disease $(21,23)$.

Spatial autocorrelation. For the 3 years of data, significant $\hat{r}_{1}$ values were found in less than half of the data sets, with $30.8,44.2$, and $30.7 \%$ of the 52 data sets showing significant positive firstorder correlations for blight, spot, and total disease, respectively, based upon correlation coefficients exceeding the bounds of their approximate $95 \%$ confidence intervals (calculated as $1.645 / \sqrt{ } N$ ). Thus, results indicate that disease clusters extended beyond the borders of the sampling units in these data sets. In data sets exhibiting significant spatial correlation, the significance typically did not extend beyond the first spatial lag $\left(\hat{r}_{1}\right)$. That is, $\hat{r}_{2}, \hat{r}_{3}$, and higherorder correlations were not significantly different from 0 . In general, spatial correlation was lowest for leaf blight and highest for leaf spot, with total disease being intermediate (Table 1). There was no general evidence of a particular type of autoregressive or moving average process $(37,38)$ for the disease incidence data, even when there was identified spatial autocorrelation.

SADIE. The SADIE program was able to calculate distance to regularity $\left(D_{\mathrm{r}}\right)$ and the distribution of the distance to regularity for randomized arrangements of the sampling-unit counts for all data sets. Magnitude of $I_{\mathrm{a}}$ values for blight, spot, and total disease were very similar across all data sets, with high similarity of all these statistics, except for a lower maximum $I_{\mathrm{a}}$ for blight (Table 1). In general, SADIE was able to detect spatial association in approximately the same percentage of data sets, $34.6,42.3$, and $32.7 \%$ for leaf blight, leaf spot, and total disease data sets, respectively, as did autocorrelation analysis.

The meaning of a large spatial association can be seen in example field E (Fig. 1; Table 2), in which $I_{\mathrm{a}}$ for both blight and spot was large (Table 1). Here, neighboring sampling units had, on average, a similar number of diseased leaflets.

Jaccard similarity analyses. At the leaflet level, values of the Jaccard index for the 52 data sets were positively skewed, with half the values falling below 0.044 (Fig. 2A). Twenty-five percent were above 0.068 (the third quartile). At the sampling-unit level, there was a larger range of Jaccard values than that at the leaflet level, and these values had less of a positive skewness. Half the Jaccard values fell below 0.24 , and $25 \%$ were above 0.49 (Fig. 2C). Higher values of the Jaccard index at the leaflet level corresponded with higher levels of Jaccard at the sampling-unit level (Fig. 2E) across the 52 data sets. That is, association (dissociation) at the leaflet level corresponded to association (dissociation) at the sampling-unit level, although the magnitude was higher for the latter.

Values of the Jaccard index generally increased with the joint increase of leaf blight and leaf spot (Fig. 3A and D). Thus, the larger Jaccard values at the sampling-unit level compared with the leaflet level (Fig. 2A and C) could be attributed to the larger disease incidence values of the former (Table 1). At the leaflet level, the Jaccard index increased somewhat with disease heterogeneity $\left(\hat{\theta}_{x}\right.$ or $\hat{\theta}_{y}$ ) (Fig. 3B), although there was no a priori expectation

TABLE 1. Minimum (Min), maximum (Max), and the three quartiles $\left(\mathrm{Q}_{1}\right.$, Median, and $\left.\mathrm{Q}_{3}\right)$ of the estimated beta-binomial parameters $\hat{p}_{t}($ expected probability of a diseased leaflet) and $\hat{\theta}$ (heterogeneity of disease), plus first-order autocorrelation coefficient $\left(\hat{r}_{1}\right)$, SADIE index of aggregation $\left(I_{\mathrm{a}}\right)$ for individual diseases, and incidence of diseased sampling units $\left(\hat{p}_{s u}\right)$ for leaf blight, leaf spot, and total disease (blight or spot) in 52 data sets collected from 1996 through 1998

\begin{tabular}{|c|c|c|c|c|c|c|c|c|c|c|c|c|c|c|c|}
\hline \multirow[b]{2}{*}{ Statistic } & \multicolumn{5}{|c|}{ Blight } & \multicolumn{5}{|c|}{ Spot } & \multicolumn{5}{|c|}{ Total disease } \\
\hline & $\hat{p}_{t}$ & $\hat{\theta}$ & $\hat{r}_{1}$ & $I_{\mathrm{a}}$ & $\hat{p}_{s u}$ & $\hat{p}_{t}$ & $\hat{\theta}$ & $\hat{r}_{1}$ & $I_{\mathrm{a}}$ & $\hat{p}_{s u}$ & $\hat{p}_{t}$ & $\hat{\theta}$ & $\hat{r}_{1}$ & $I_{\mathrm{a}}$ & $\hat{p}_{s u}$ \\
\hline Min & 0.005 & 0.01 & -0.21 & 0.52 & 0.040 & 0.001 & 0.00 & -0.18 & 0.49 & 0.013 & 0.027 & 0.03 & -0.19 & 0.50 & 0.224 \\
\hline $\mathrm{Q}_{1}$ & 0.059 & 0.10 & 0.00 & 0.90 & 0.394 & 0.017 & 0.11 & 0.01 & 1.06 & 0.118 & 0.131 & 0.12 & 0.03 & 0.87 & 0.552 \\
\hline $\mathrm{Q}_{3}$ & 0.321 & 0.20 & 0.22 & 2.04 & 0.888 & 0.240 & 0.36 & 0.31 & 2.04 & 0.802 & 0.508 & 0.24 & 0.24 & 1.97 & 0.986 \\
\hline Max & 0.613 & 0.35 & 0.51 & 3.67 & 1.000 & 0.812 & 1.06 & 0.85 & 5.46 & 1.000 & 0.862 & 0.60 & 0.50 & 5.08 & 1.000 \\
\hline
\end{tabular}

TABLE 2. Estimated beta-binomial parameters $\hat{p}_{t}$ (expected probability of a diseased leaflet) and $\hat{\theta}$ (heterogeneity of disease), plus SADIE index of aggregation $\left(I_{\mathrm{a}}\right)$ for individual diseases and various measures of association for leaf blight, leaf spot, and total disease for the six example fields of Figure 1

\begin{tabular}{|c|c|c|c|c|c|c|c|c|c|c|c|c|c|c|c|}
\hline \multirow{3}{*}{$\begin{array}{l}\text { Figure } \\
\text { reference }\end{array}$} & \multicolumn{9}{|c|}{ Disease } & & & & & & \\
\hline & \multicolumn{3}{|c|}{ Blight $(x)^{\mathrm{a}}$} & \multicolumn{3}{|c|}{ Spot $(y)^{\mathrm{a}}$} & \multicolumn{3}{|c|}{$\operatorname{Total}(z)^{\mathrm{a}}$} & \multicolumn{6}{|c|}{ Measures of Association ${ }^{\mathrm{b}}$} \\
\hline & $\hat{p}_{t}$ & $\hat{\theta}$ & $I_{\mathrm{a}}$ & $\hat{p}_{t}$ & $\hat{\theta}$ & $I_{\mathrm{a}}$ & $\hat{p}_{t}$ & $\hat{\theta}$ & $I_{\mathrm{a}}$ & $J_{t}$ & $s_{J}$ & $\bar{J}_{t,(\text { ran })}$ & $\hat{\rho}_{0}$ & $I$ & $J_{s u}$ \\
\hline $\mathrm{B}$ & 0.27 & 0.27 & 3.32 & 0.18 & 0.16 & 1.91 & 0.38 & 0.23 & 3.41 & 0.19 & 0.030 & 0.16 & $0.42 * \mathrm{c}$ & 1.55 & 0.75 \\
\hline $\mathrm{C}$ & 0.17 & 0.12 & 1.18 & 0.28 & 0.11 & 2.10 & 0.42 & 0.09 & 1.01 & $0.06^{*}$ & 0.013 & 0.10 & -0.15 & $0.36^{*}$ & 0.69 \\
\hline D & 0.47 & 0.35 & 0.95 & 0.56 & 0.33 & 1.81 & 0.81 & 0.15 & 1.59 & 0.27 & 0.025 & 0.28 & $-0.48^{*}$ & $0.45^{*}$ & 0.94 \\
\hline $\mathrm{E}$ & 0.40 & 0.18 & 3.67 & 0.34 & 0.39 & 2.60 & 0.64 & 0.14 & 1.10 & 0.16 & 0.024 & 0.18 & $-0.35^{*}$ & 0.43 & 0.76 \\
\hline
\end{tabular}

a Symbol used for leaf blight, leaf spot, and total (blight or spot) disease in text.

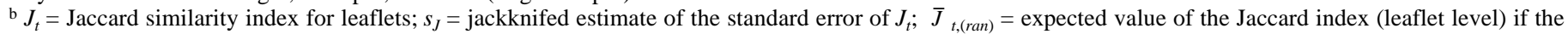
two diseases were distributed independently, but with both individually aggregated in fields; $\hat{\rho}_{0}=$ Spearman's rank correlation coefficient of the incidence of the two diseases in the same sampling units; and $I=$ index of covariation of two diseases based on the SADIE procedure (33,35) for two species.

c $*=$ Significant at $P \leq 0.05$. Test results only shown for association statistics.

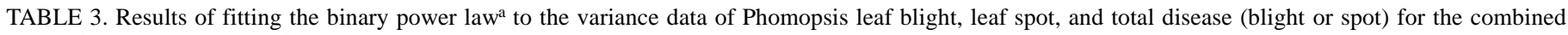
years of 1996 and 1997 and for 1998

\begin{tabular}{|c|c|c|c|c|c|c|}
\hline & \multicolumn{3}{|c|}{1996 and 1997} & \multicolumn{3}{|c|}{1998} \\
\hline & $\ln (\hat{A}) \pm \mathrm{SE}$ & $\hat{\beta} \pm \mathrm{SE}$ & $R^{2}$ & $\ln (\hat{A}) \pm \mathrm{SE}$ & $\hat{\beta} \pm \mathrm{SE}$ & $R^{2}$ \\
\hline Blight & $1.00 \pm 0.039$ & $1.17 \pm 0.040$ & 0.97 & $0.89 \pm 0.072$ & $1.19 \pm 0.080$ & 0.89 \\
\hline Spot & $1.28 \pm 0.085$ & $1.21 \pm 0.049$ & 0.97 & $1.32 \pm 0.070$ & $1.23 \pm 0.057$ & 0.95 \\
\hline Total & $1.05 \pm 0.086$ & $1.07 \pm 0.091$ & 0.87 & $0.91 \pm 0.114$ & $1.31 \pm 0.115$ & 0.83 \\
\hline
\end{tabular}

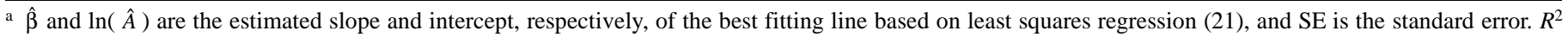
is the coefficient of determination. 
that the Jaccard index should increase with larger values of $\theta$. This may reflect the fact that $\hat{\theta}$ is related to $\hat{p}$ for these two diseases, because $\hat{\beta}$ of the binary power law was greater than 1 (13) (Table $3)$. Values of the Jaccard index did not follow any systematic trend with spatial autocorrelations $\left(\hat{r}_{1}\right)$ or SADIE indices $\left(I_{\mathrm{a}}\right)$ of the individual diseases (data not shown).

At the leaflet level, only four data sets had observed Jaccard values significantly different from the calculated expected value under independence ( $\left.\bar{J}_{t,(\text { ran })}\right)$ according to a standard normal test (equation 3 ). Given that each test had a 0.05 probability of rejecting the null hypothesis of independence when the data were independent, one would expect to find approximately three data sets with significant results, even if there was no association of the diseases. In general, results indicate that the two diseases predominantly occurred independently of each other at the leaflet level. When the observed Jaccard values were regressed onto their respective $\bar{J}_{t,(\text { ran })}$ values, estimates of the slope were slightly less than 1 , suggesting that, over the range of conditions studied, leaf blight and leaf spot were slightly dissociated (Fig. 4A). In the vast majority of cases, however, $J_{t} \pm s_{J}$ overlapped the $\bar{J}_{t,(\text { ran })}$ value for that data set (Fig. 4A). The $J_{t}$ s calculated directly from the observed data fell about midway between the maximum and minimum possible values of $J_{t}\left(J_{t,(\max )}, J_{t,(\min )}\right)$ for the individual data sets. In general, the separation of $J_{t,(\max )}$ and $J_{t,(\min )}$ increased with increasing $J_{t,(\text { ran })}$ or $J_{t}$. Figure $1 \mathrm{~A}, \mathrm{~B}, \mathrm{D}$, and E depicts example cases in which $J_{t}$ was not significantly $(P>0.05)$ different from $\bar{J}_{t,(\mathrm{ran})}$, and Figure $1 \mathrm{C}$ and $\mathrm{F}$ depicts cases in which $J_{t}$ was significantly $(P<$ $0.05)$ smaller than $\bar{J}_{t,(\text { ran })}($ Table 2$)$.
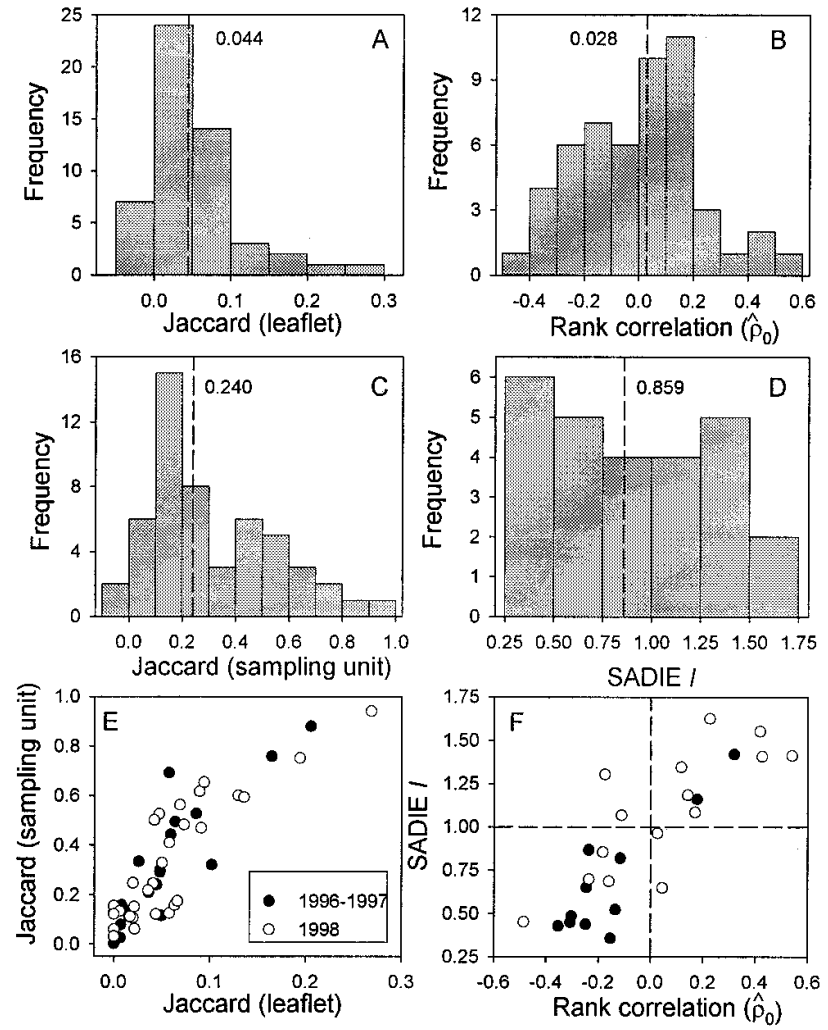

Fig. 2. Frequency distributions of $\mathbf{A}$, Jaccard similarity index at the leaflet scale; B, Spearman's rank correlation coefficient; $\mathbf{C}$, Jaccard similarity index at the sampling-unit level; and D, SADIE-based index of covariation; and the relationship between $\mathbf{E}$, Jaccard similarity indices at the leaflet and samplingunit levels and F, Spearman's rank correlation and SADIE's index of covariation for 52 independent field assessments of Phomopsis leaf blight and strawberry leaf spot epidemics for 1996 to 1998. A and C, The first bar represents the number of data sets in which the Jaccard index was 0 (no leaflets or sampling units had both diseases). A to $\mathbf{D}$, Median values are shown as broken lines and numerical values are provided. F, Reference lines representing independence $\left(I=1 ; \hat{\rho}_{0}=0\right)$ are shown as broken lines.
At the sampling-unit level, no Jaccard value $\left(J_{s u}\right)$ was significantly different from its corresponding $\bar{J}_{s u,(r a n)}$, even though the Jaccard values were larger at the sampling-unit level than at the leaflet level (Fig. 2A and C). This suggested that these two diseases occurred independently of each other at the level of the sampling unit, at least in terms of the binary classification of sampling units for the presence of the two diseases. A regression of $J_{s u}$ onto $\bar{J}_{s u(\text { ran })}$ produced a line with a slope of nearly 1 (Fig. 4B). Most observed values were on or very close to the $\bar{J}_{s u,(\text { ran })}$ line. At the sampling-unit level, the maximum and minimum envelopes $\left(J_{s u,(\min )}\right.$ and $\left.J_{s u,(\max )}\right)$ were very narrow relative to the magnitude of the jackknifed standard errors of the individual data sets (Fig. 4B), making it difficult to declare any possible Jaccard value significant and to interpret the meaning of the observed Jaccard values. The narrow envelopes may be related to the high disease incidence at the sampling-unit level for at least one of the two diseases. For instance, $\hat{p}_{s u, x}$ and $\hat{p}_{s u, y}$ were 0.80 or higher in $75 \%$ of the data sets (Table 1, third quartile). As incidence approaches 1, there is little possible variation in the Jaccard index between the maximum and minimum values under any degree of association.

Covariation analyses-Correlations and SADIE. Rank correlations between blight and spot incidence in the same sampling units $\left(\hat{\rho}_{0}\right)$ ranged from -0.48 to 0.54 , with a median of 0.03 (Fig. 2B). There was little skewness in the distribution of values across fields. Of the $52 \hat{\rho}_{0}$ values, 11 were significantly different from 0 $(P<0.05)$, four being larger than 0 , and seven smaller. Analysis of the majority of data sets indicated that the two diseases varied independently of each other. Significant cross-correlations (correlations at spatial lags greater than 0 ) were typically associated only with significant $\hat{\rho}_{0}$ s. In general, cross-correlations were not significant beyond $\rho_{+1}$ and $\rho_{-1}$, i.e., the first-order correlation between neighboring sampling units (data not shown).

Figure 1B depicts an example case with significant positive correlation, and Figure 1D and E depicts cases with negative correlation (Table 2). For fields with the negative $\hat{\rho}_{0}$, one can see that high numbers of leaflets with blight symptoms were associated with low numbers of leaflets with spot symptoms (Fig. 1D and E). For the field example with positive $\hat{\rho}_{0}$ (Fig. 1B), there was some tendency for the numbers of diseased leaflets to be either jointly high or jointly low for spot and blight, especially in the second half of the field. However, the trend was visually weaker than for the examples with negative $\hat{\rho}_{0}$.

The SADIE-based test for disease covariation, conditioned on the spatial pattern of the individual diseases (33), could only be completed on 26 of the 52 data sets. The primary reason for the failure of the test was the inability of the SADIE algorithm to produce a randomization of the original data with $D_{\mathrm{r}}$ and $\delta$ values close to the original for one of the diseases. This problem could be alleviated for some data sets by specifying less stringent matching criteria (33). Results shown are based on the nominal criteria for performing the procedure. Including the results from use of the less stringent requirements did not change the trends and relationships shown here (W. W. Turechek, unpublished data).

The distribution of the SADIE index, $I$, across the 52 fields was fairly uniform, with about the same number of values above and below 1 (Fig. 2D). Of the 26 data sets with calculated statistics, four showed significant negative covariation $(P<0.05)$ and none showed significant positive covariation. Figure $1 \mathrm{C}$ and D illustrates fields with significant negative covariation as determined by the SADIE-based procedure (Table 2). There was a linear relationship between $I$ and $\hat{\rho}_{0}$ (Fig. $2 \mathrm{~F}$ ), indicating that rank correlations and SADIE measured similar aspects of disease covariation. However, the results of the significance tests were not always the same. For instance, although $I$ for field B (Fig. 1B) was above 1, it was not significantly so (Table 2 ).

There was no obvious relationship between incidence of leaf blight, incidence of leaf spot, and measures of covariation such as $\hat{\rho}_{0}$ and $I$ (Fig. 3C). Likewise, there was no relationship between 
measures of spatial pattern of individual diseases $\left(\hat{r}_{1}, I_{\mathrm{a}}\right)$ and measures of covariation of the two diseases, such as $\hat{\rho}_{0}$ and $I$ (data not shown).

Field-level associations and total disease. Rank correlations indicated that there was no relationship $(P>0.05)$ between incidence of blight $\left(\hat{p}_{t, x}, \hat{p}_{s u, x}\right)$ and incidence of spot $\left(\hat{p}_{t, y}, \hat{p}_{s u, y}\right)$ at the field level for those fields with both diseases, regardless if incidence was assessed at the leaflet or sampling-unit level (Fig. $5 \mathrm{~A}$ and $\mathrm{B}$ ). No one disease was consistently higher or lower than the other at either hierarchical scale. Rank correlations also showed that there was no relationship between $\hat{\theta}_{x}$ and $\hat{\theta}_{y}$ or between $\hat{r}_{1, x}$ and $\hat{r}_{1, y}$ (Fig. 5C and D). This can also be seen by the overlap of the frequency distributions of these statistics (Table 1). Because of consistent results between SADIE and autocorrelations (Fig. 2F), graphs are not shown for $I_{\mathrm{a}}$.

The Jaccard index of similarity at the field scale (in which a field was coded as diseased if one or more leaflets had disease symptoms) was $0.42(a=52, b=72$, and $c=0)$. This value was smaller than $33 \%$ of the Jaccard values at the sampling-unit level for the 52 data sets with both diseases. Because blight was present in all fields, randomizations could not be performed, in that the same value of $J$ would be obtained with any randomization. Thus, no statistical test could be performed for independence.

Predicted incidence of total disease under independence $\left(\hat{p}_{t, z, \text { (ind })}\right)$ was very close to the observed values of total disease incidence $\left(\hat{p}_{t, z}\right.$, the estimated parameter of the beta-binomial for total disease) (Fig.
6A). The median value of $\hat{p}_{t, z}-\hat{p}_{t, z \text { (ind })}$ over the 52 data sets was 0.00 , with a range of -0.025 to 0.054 . Most values of observed $\hat{p}_{t, z}$ were about midway between the maximum and minimum possible total disease incidence values $\left(\hat{p}_{t, z_{1}(\max )}\right.$ and $\left.\hat{p}_{t, z_{1}(\min )}\right)$, given the observed incidence and heterogeneity of the individual diseases (Fig. 6A).

Regression results for the relationship between $\hat{p}_{t, z,(i n d)}$ and $\hat{p}_{t, z}$ are also shown in Figure 6A (solid line). The estimates and their corresponding standard errors (SE) of the slope and intercepts parameters were $1.02(\mathrm{SE}=0.008)$ and $-0.004(\mathrm{SE}=0.003)$, respectively, with a coefficient of determination of $99.7 \%$. The slope estimate was slightly larger than $1(P=0.001)$, indicating that there was a trend for these two diseases to be slightly dissociated at the field level. However, with a slope of only 0.02 units from 1.00 , this trend was very small. Results from the regressions of $\hat{p}_{t, z,(\max )}$ or $\hat{p}_{t, z,(\min )}$ on $\hat{p}_{t, z,(\text { ind })}$ are shown as broken lines (Fig. 6A). As expected, estimated slopes were above and below the estimated slope for the observed value (Fig. 6A).

A way of considering heterogeneity of total disease at the field scale was to compare $\hat{\theta}_{z}$ with an estimate of $\hat{\theta}_{z}$, assuming that leaf blight and leaf spot were distributed independently of each other ( $\hat{\theta}_{z, \text { (ind) }}$ ) (equation 5). There was a linear relationship between $\hat{\theta}_{z}$ and $\hat{\theta}_{z, \text { ind })}$ (Fig. 6B), with many of the points falling on or around the line with the slope of 1 and intercept of 0 (1:1 line). Heterogeneity of observed total disease incidence, as measured by $\hat{\theta}_{z}$ of the beta-binomial distribution, was generally intermediate between the observed heterogeneity of the individual diseases ( $\hat{\theta}_{x}$ and $\left.\hat{\theta}_{y}\right)$.
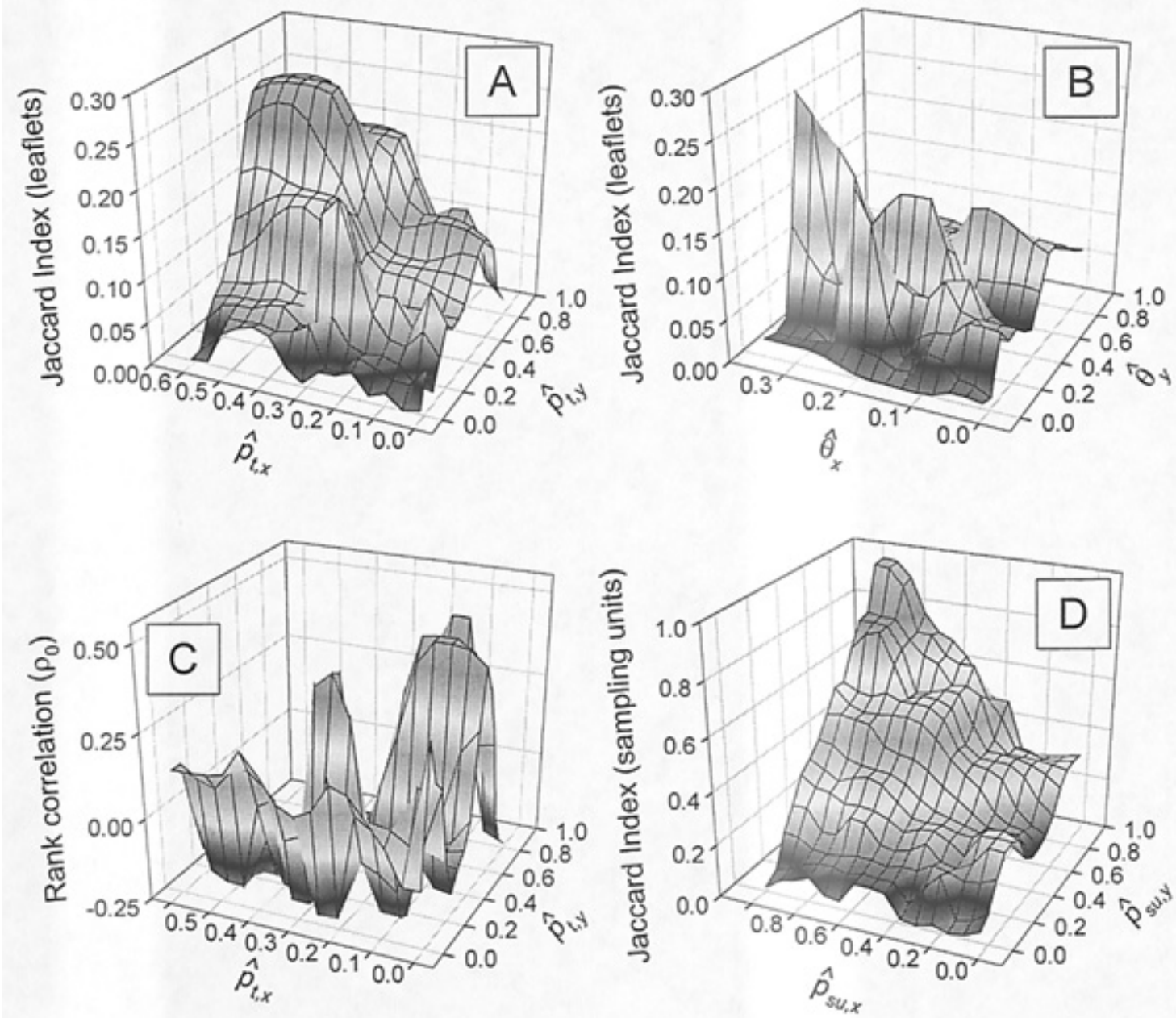

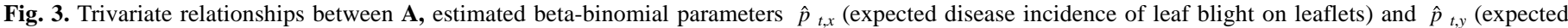

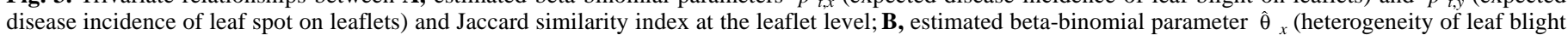

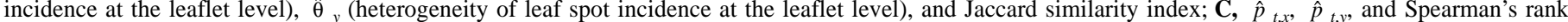

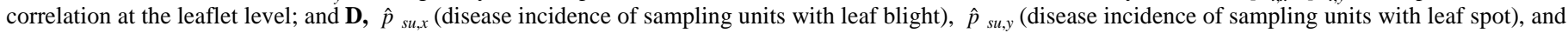

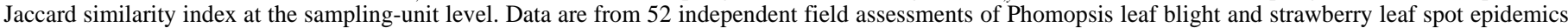
made over 3 years, 1996 to 1998. Surface mesh plots were produced using Sigma Plot (3D-interpolator with weight seven). 


\section{DISCUSSION}

Association between diseases is manifested, in part, in the spatial patterns of the individual diseases in fields (33). Thus, quantifying disease associations must be done with consideration of their spatial patterns. Leaf blight and leaf spot of strawberry were qualitatively similar, in that incidences of both were overdispersed relative to the binomial distribution, and both were well described by the beta-binomial distribution in the same fields. We have also shown that Phomopsis leaf blight and strawberry leaf spot were distributed independently of each other or were very weakly dissociated at the leaflet and sampling-unit scales, in terms of their co-occurrence and covariation, based on the Jaccard similarity index, data randomizations, rank correlations, and a new SADIE-
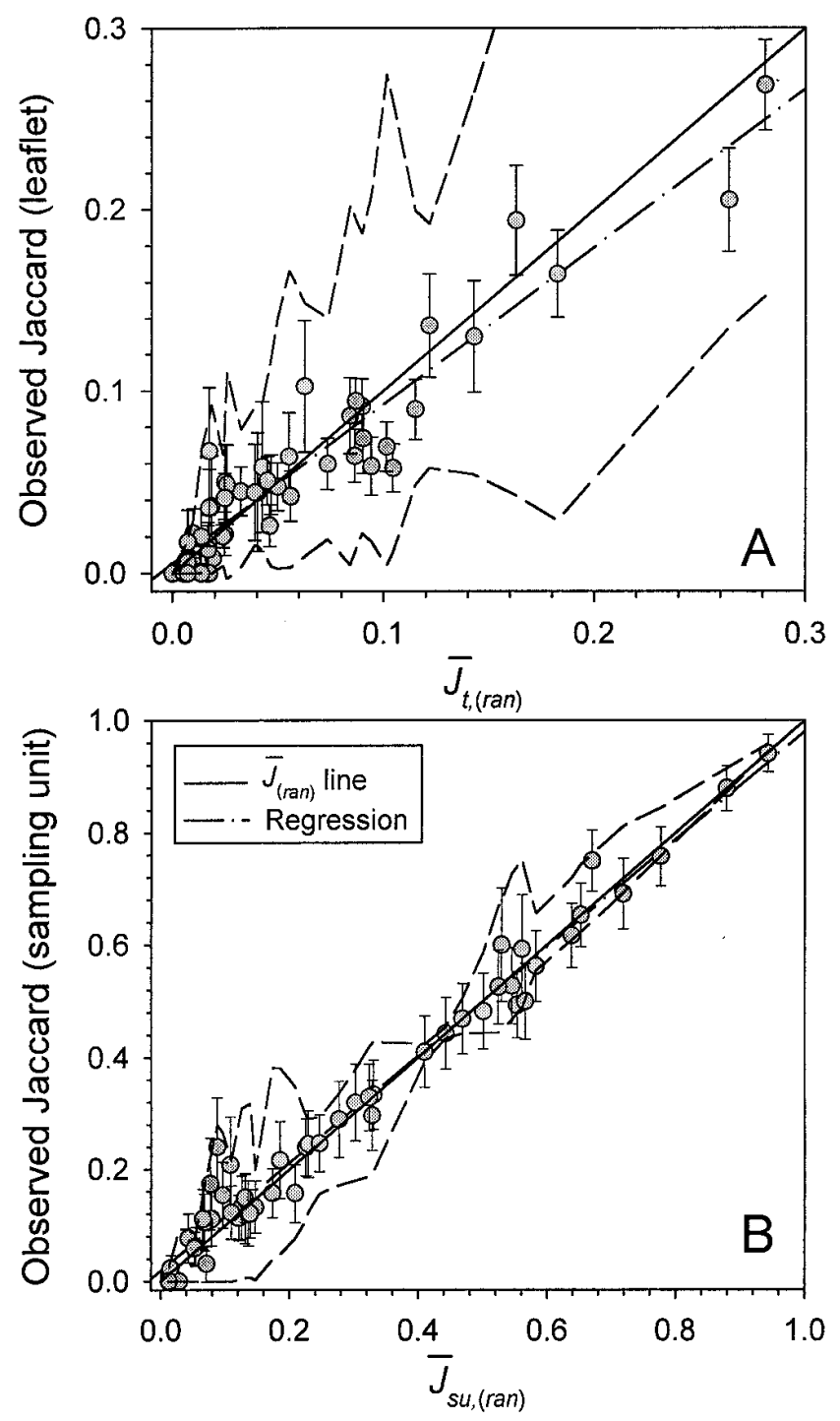

Fig. 4. Relationship between the average randomized value of the Jaccard similarity index (under the assumption of independence of the leaf spot and leaf blight diseases) conditioned on the observed incidence and heterogeneity of each disease, and the observed Jaccard similarity index at the $\mathbf{A}$, leaflet level $\left(J_{t}\right)$ and $\mathbf{B}$, sampling-unit $\left(J_{s u}\right)$ level (circles). Standard errors were estimated using the jackknife procedure and are shown as the vertical bars. Maximum and minimum Jaccard envelopes (maximum and minimum possible Jaccard index for each data set) are shown as the broken lines above and below the data points, respectively. Envelopes were smoothed using the LOcally WeightEd Scatter plot Smoother (LOWESS) procedure in Sigma Plot, with a weight factor of 0.12 . The independence line is shown as the solid line, and least-squares fit to the data is shown as the broken line. Points are from 52 data sets of Phomopsis leaf blight and leaf spot epidemics of strawberry made over 3 years, 1996 to 1998. based procedure. Covariation of disease incidence at the field scale was also shown to be uncommon based on the lack of significant relationship between mean leaflet disease incidence for the two diseases (Fig. 5A and B).

The randomization test developed here (Jaccard adaptation) determined the expected value of the Jaccard similarity index under the assumption of independence and tested the hypothesis of no association-specifically, no co-occurrence-while accounting for the spatial characteristics $\left(\hat{\theta}_{x}, \hat{\theta}_{y}, \hat{r}_{1, x}\right.$, and $\left.\hat{r}_{1, y}\right)$ of the two individual diseases. Furthermore, the test assigned a statistical meaning to the observed Jaccard value that was absent without the randomizations; that is, the randomizations could be used to show how far the observed Jaccard index was from its value under independence, given the observed distributional properties of leaf blight and leaf spot. In most cases, the observed statistic was very close to the value for independence.

Although we chose the Jaccard index, the procedures developed here for aggregated diseases are applicable to the many indices used to test for pairwise species associations, such as the Dice and Ochiai statistics (17). It should be noted that these statistics are not very informative when incidence of one of the diseases is very high (close to 1), such as at the field scale-in which a field is considered diseased if one leaflet is infected-because there is virtually no range in possible Jaccard values with randomizations. The impact of high incidence can be seen by the maximum and minimum envelopes of Jaccard values at the sampling-unit level (Fig. 4B), in which $\hat{p}_{s u, x}$ and $\hat{p}_{s u, y}$ (proportion of diseased sampling units) were both high (Table 1). The high incidence can be attributed to the relationship between incidence at the leaflet and sampling-unit scales, which typically follows a saturation-type curve $(15,42)$. Thus, incidence at the leaflet level (either spot or blight) does not have to be very high before most of the sampling units are diseased. As the proportion of diseased sampling units approaches 1, the difference between maximum and minimum possible Jaccard
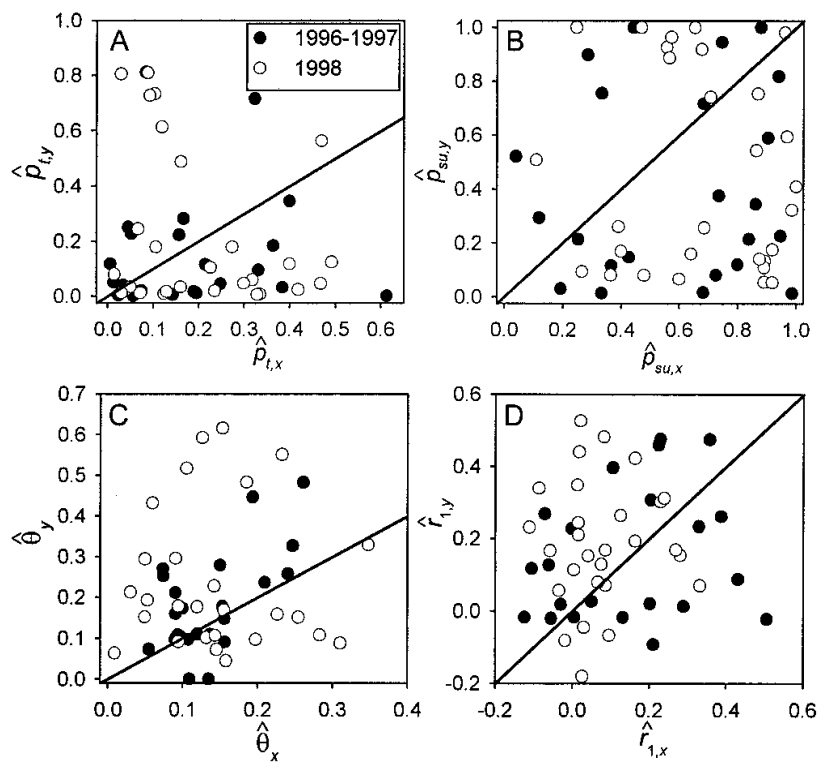

Fig. 5. Bivariate relationships between A, estimated beta-binomial parameters $\hat{p}_{t, x}$ (expected disease incidence of leaf blight on leaflets) and $\hat{p}_{t, y}$ (expected disease incidence of leaf spot on leaflets); $\mathbf{B}, \hat{p}_{s u, x}$ (disease incidence of sampling units with leaf blight) and $\hat{p}_{s u, y}$ (disease incidence of sampling units with leaf spot); $\mathbf{C}$, estimated beta-binomial parameter $\hat{\theta}_{x}$ (heterogeneity of leaf blight incidence on leaflets) and $\hat{\theta}_{y}$ (heterogeneity of leaf spot incidence on leaflets); and $\mathbf{D}$, estimated first-order correlation of leaf blight $\left(\hat{r}_{1, x}\right)$ and estimated first-order correlation of leaf spot $\left(\hat{r}_{1, y}\right)$. The one-to-one lines are shown as solid lines. A, B, C, and D, Rank correlations for the bivariate relationships were $-0.02,-0.02,0.21$, and 0.11 , respectively. Points are from 52 data sets of Phomopsis leaf blight and leaf spot of strawberry epidemics made over 3 years, 1996 to 1998. 
index values approaches 0 for a given data set, hence making it difficult to declare any value of the Jaccard significantly different from 0 , even if ecological association exists.

A possible drawback to the test based on randomizations is that the procedure used here relies on the assumption that the calculated Jaccard index follows a normal distribution (6). With the large data sets analyzed here $(N>50)$, this is probably a reasonable assumption. With smaller data sets (e.g., $N<30$ ), this test is not recommended. An alternative approach, which would use the same randomizations as done here, would be to simply calculate the proportion of randomizations with Jaccard values larger and smaller than the observed index, and use these proportions to calculate the appropriate $P$ value for the test.

When measuring association, it is important to consider the spatial scale at which the data were collected (27). Because of the hierarchical nature of the data, five spatial scales were available to examine association: the leaflet, sampling-unit, field, farm, and regional scales. Although the lowest three scales were the primary focus of this research, Jaccard indices can, in fact, be constructed at each of the five spatial hierarchical scales for comparison purposes. At the leaflet scale, pooled over all data sets (not just those with both diseases present), 1,277 of the 17,037 diseased leaflets (leaflets with blight or spot) had symptoms of both diseases, giving a Jaccard index of 0.075 ; at the sampling-unit scale, 891 sampling units had both diseases from the 2,638 that had either, giving a Jaccard index value of 0.38 ; at the field scale, the Jaccard index was $52 / 124=0.42$. At the farm scale, the Jaccard index was $17 / 27=$ 0.63 ; and at the regional scale, the Jaccard index was $7 / 9=0.78$. The interpretation of the indices is straightforward, representing the probability of encountering both diseases in the same unit for those units with at least one disease. For example, at the leaflet scale, one had approximately a $7.5 \%$ chance of finding a leaflet with symptoms of both diseases among those leaflets with symptoms of at least one disease. As one moves from lower to higher levels in the hierarchy, the probability of encountering both diseases must remain the same or increase. Although the Jaccard values were large at the higher scales, it is not possible to test these for significance, because leaf blight was present in every data set. As with the field-scale tests, there was no variation to Jaccard values under randomizations.

Leaf blight and leaf spot of strawberry require similar climatic conditions for disease development $(8,18)$. Thus, at the regional scale, it is reasonable to conclude that any positive co-occurrence of the two diseases is a result, at least in part, of large-scale climate patterns. Since all of the farms were located in favorable regions for the two diseases, one would expect the level of association to be relatively high at the farm scale, and quite possibly the field level as well. However, at lower levels in the spatial hierarchy, meso- and micro-environmental processes, micro-geographical features, patchiness of the crop, and human intervention induces ecological heterogeneity into the system with regard to favorableness for disease development $(16,27)$. At the farm and field levels, farming practices (e.g., fungicide applications, cultivar selection, and watering practices), local weather phenomena, and local physical features may have more of a direct influence on disease association than do the factors influencing associations at much higher scales.

The general lack of significant co-occurrence, as determined with the Jaccard adaptation, between leaf blight and leaf spot at the leaflet or sampling-unit scales, suggests that some aspects of the biology of these pathogens may differ. Since P. obscurans and $R$. brunnea have similar temperature and leaf wetness requirements for disease development $(8,18)$ and have similar disease cycles, their independence within fields cannot necessarily be attributed to different environmental conditions required for disease development. However, one of the primary mechanisms behind spatial pattern formation, and quite possibly disease association, is mode of spore dispersal $(10,29,48)$. The spatial patterns of these two individual diseases are quantitatively different, in terms of the magnitude of $\hat{\theta}$ (for within-sampling-unit patterns) and $\hat{r}_{1}$ (for between-samplingunit patterns) (42,43) (Table 1). P. obscurans is splash-dispersed (18), and Turechek and Madden (43) presented an argument that $R$. brunnea may have a wind-dispersed component, even though there are no studies showing this. Thus, the putatively different modes of spore dispersal, which were suggested to partially contribute to the quantitatively different spatial patterns of the two diseases (43), may have influenced the degree of association.

The lack of co-occurrence of the diseases found in this study does not imply that the two pathogens do not interact at another (lower) scale. For example, spores of the two pathogens could compete for infection sites on leaflets (41). Any dissociation of inoculum
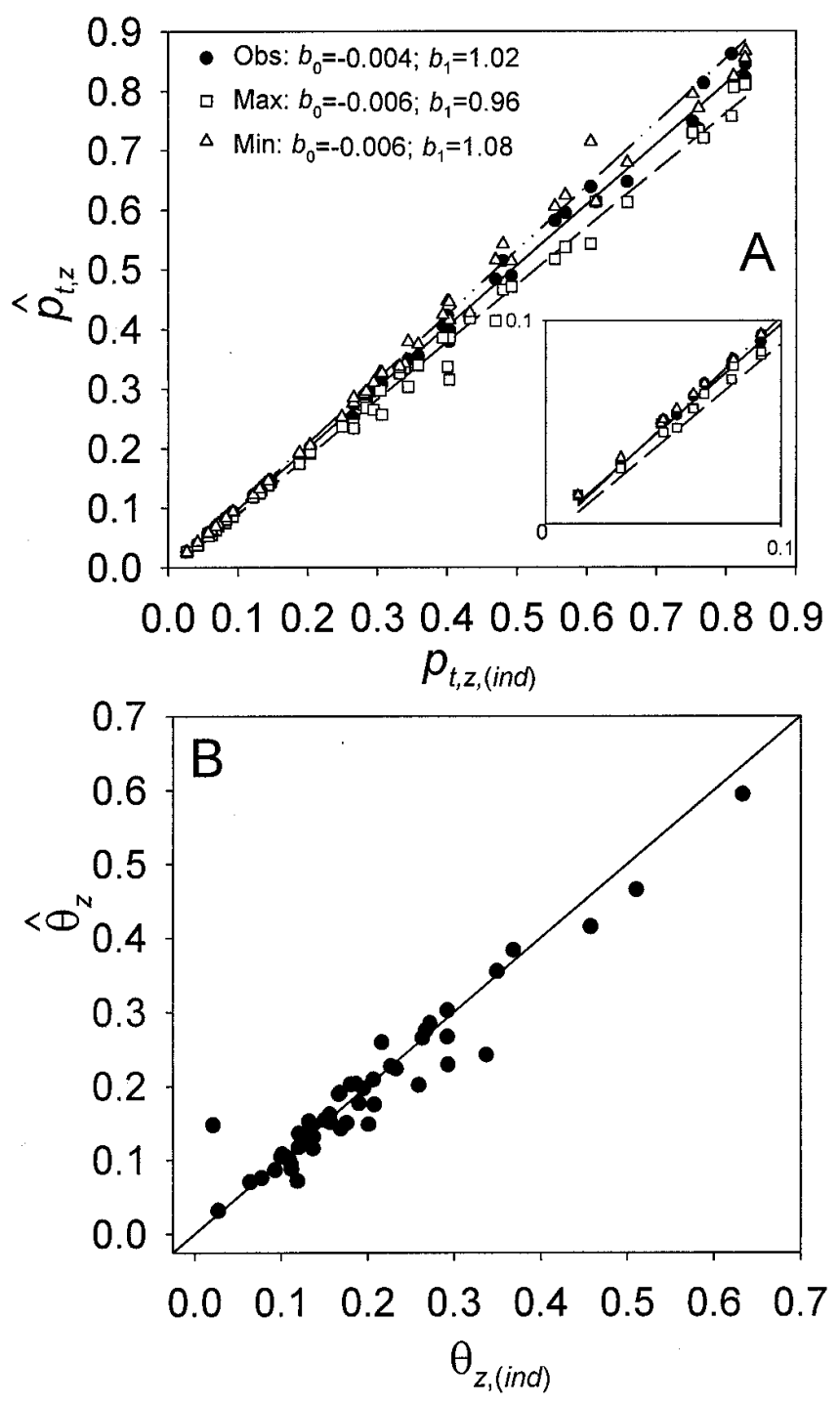

Fig. 6. A, Observed total disease incidence (leaf blight or leaf spot) $\left(\hat{p}_{t, z} ;[\mathbf{\bullet}]\right)$, maximum possible total disease incidence $\left(\hat{p}_{t, z,(\max )}[\Delta]\right.$, which corresponds to the minimum possible association), and minimum possible total disease incidence ( $\hat{p}_{t, z,(\min )}[\square]$, which corresponds to the maximum possible association) in relation to the estimated total disease incidence with no association between leaf blight and leaf spot at the leaflet scale $\left(\hat{p}_{t, z,(i n d)}\right)$. Maxima and minima are based on the observed incidence and heterogeneity of individual diseases. Least-squares fit to the observed, maximum, and minimum values of $\hat{p}_{t, z}$ are shown, with $b_{0}$ and $b_{1}$ representing the intercepts and slopes, respectively. 'Max' and 'Min' refer to association, not total incidence. Inset: enlargement of the graph between incidence values of 0 and 0.1 . B, Relationship between estimated beta-binomial heterogeneity parameter for total disease incidence, $\hat{\theta}_{z}$, and a moment-like estimate of heterogeneity of total disease incidence based on assumed independence of leaf blight and leaf spot and a weighted combination of $\hat{\theta}_{x}$ and $\hat{\theta}_{y}, \hat{\theta}_{z, \text { (ind) }}$ (equation 5). Solid lines represent 1:1 correspondence. Points are from 52 data sets of Phomopsis leaf blight and leaf spot of strawberry epidemics made over 3 years, 1996 to 1998. 
or disease at the cellular level here could, in principle, negate any association at higher scales at the farm or region. Furthermore, because pathogen (or species) density has been recognized as a contributing factor to both inter- and intraspecific competition $(11,30,31,39)$, the density of either pathogen or its propagules in the soil, on debris, or on plant parts may have also had an influence on the lack of association. No information is known about the pattern of soil- or debris-borne inoculum of these two pathogens in Ohio or elsewhere.

In addition to the association analysis for co-occurrence, rank correlations and the SADIE-based approach were used to quantify covariation at multiple scales. The SADIE approach $(33,35)$ has the advantages of being explicitly designed for count (rather than continuous) data, conditioning results on the spatial pattern of the individual diseases, and detecting correlations in disease incidence in neighboring sampling units (which is also possible with crosscorrelation analysis) as well as within sampling units. Unfortunately, with the SADIE-based procedure, it is not possible to directly determine whether significant covariations were due to correlations within the same sampling units or neighboring sampling units. With cross-correlation analysis, one is able to explicitly determine these separately, but this may not be easy in studies in which data are from two-dimensional grids rather than from transects. The agreement in results between these two procedures was very good (Fig. $2 \mathrm{E})$. However, the null hypothesis of independence was rejected more often for rank correlation than for the SADIE-based procedure. This may be a reflection of higher false-positive rates for rank correlation, because this method fails to account for the heterogeneity and spatial pattern of the two diseases $(3,33,46)$. Nevertheless, in agreement with the Jaccard adaptation analyses of association, no positive or negative covariation was found for most data sets. It should be noted that significant covariation was found in only 11 data sets with rank correlations, and the SADIE procedure produced significant results for four of these. The SADIE procedure was not appropriate for the field-level (or higher scale) analyses here, because the fields were not arranged in well-defined spatial structure $(33,35)$.

The relationship between incidence of total disease and the two component diseases provided a probabilistic means of confirming independence of spot and blight at the field level. In general, observed incidence of leaflets with any disease $\left(\hat{p}_{t, z}\right)$ was very close to predicted total disease incidence under independence $\left(\hat{p}_{t, z, \text { (ind })}\right)$ (Fig. 6A). Moreover, a regression analysis of $\hat{p}_{t, z}$ on $\hat{p}_{t, z \text {, (ind) }}$ showed evidence that leaf blight and leaf spot could be slightly dissociated at the field level, because the slope was slightly larger than 1 . This indicates that the difference between $\hat{p}_{t, z}$ and $\hat{p}_{t, z, \text { (ind) }}$ increased as $\hat{p}_{t, z}$ (and, hence, $\hat{p}_{t, x}$ and $\hat{p}_{t, y}$ ) increased (Fig. 6A), even if the individual differences were not different from 0 . Results for the regression analyses through the maximum and minimum values of total disease produced a narrow range of slope and intercept values; however, values within this range were large enough to detect departures from independence (slope different from 1), because the standard error of the estimated slope was very small. It is not clear if the slope being significantly larger than 1 has any biological meaning.

Under the assumption that leaf blight and leaf spot are distributed independently of each other and that total disease incidence could be described by the beta-binomial distribution, a momentlike estimate of $\theta_{z}$ was calculated (equation 5) based on a weighted intracluster correlation $\left(\phi_{(w t d)}\right)$ (equation 7), in which the weights were based on the magnitude of the individual disease incidences. Although equation 5 provided only an empirical approach to estimating heterogeneity of total disease incidence when the component diseases are distributed independently of each other, the predictions of $\theta_{z}\left(\hat{\theta}_{z, \text { ind })}\right)$ were very close to the observed values $\left(\hat{\theta}_{z}\right)$, providing further evidence that leaf blight and leaf spot of strawberry were distributed independently of each other.

Transect maps from six example fields show the range of conditions encountered in this study and the influence each condition had on the statistics used. Figure 1A depicts the typical case of no association or covariation, as determined by any test (Table 2, A). However, the spatial patterns of the individual diseases were not random; estimated values of $\theta$ were greater than 0 and values of $I_{\mathrm{a}}$ were greater than 1 (Table 2). Figure 1B shows an example of positive covariation, with the peaks and valleys in the incidences of the two diseases varying together overall. Figure $1 \mathrm{C}$ shows dissociation (small $J_{t}$ ) and negative covariation (as determined by $I<1$ $[P<0.05])$. Figure $1 \mathrm{D}$ and $\mathrm{E}$ shows negative covariation as determined from the rank correlation coefficient; however, only D shows negative covariation as determined by $I$ (Table 2 ). As measured by $\hat{\theta}\left(\hat{\theta}_{x}\right.$ or $\left.\hat{\theta}_{y}\right)$, the degree of heterogeneity was quite large for both individual diseases in Figure 1D, and large only for spot in Figure 1E (Table 2). The conditioning of $I$ on the degree of spatial heterogeneity (33) may have had an influence as to why similar values of $I$ produce different results of significance. Notice that high peaks of each disease occurred in different sampling units in Figure 1D. This cross-correlation can not be measured by $\hat{\rho}_{0}$ alone, but can be detected by the SADIE-based procedure. Finally, Figure $1 \mathrm{~F}$ shows dissociation as determined by the Jaccard adaptation $\left(J_{t}<\right.$ $\left.\bar{J}_{t,(\mathrm{ran})}\right)$ at the leaflet level, but no significant covariation values. This is a case in which one can clearly see how the peaks and valleys of individual disease incidence do not correspond with each other, even though there is an overall significant disassociation of diseased leaflets in the field.

In conclusion, results based on the Jaccard index, data randomizations, rank correlations, and a new SADIE-based procedure clearly demonstrated that Phomopsis leaf blight and strawberry leaf spot were, in the majority of data sets, distributed independently of each other at the leaflet, sampling-unit, and field levels in commercial strawberry fields over 3 years. The tests used for determining association depended on the characteristics being assessed (occurrence or covariation) and the hierarchical scale of the data (e.g., leaflet or field) (17). Moreover, the tests based on randomizations and the SADIE-based procedure conditioned results on the spatial patterns of leaf blight and leaf spot, thus eliminating the effects of spatial heterogeneity of the individual diseases from the association analyses. The independence between the two diseases was partly attributed to their putatively different modes of spore dispersal. Because of the independence, prediction of total disease incidence and heterogeneity of total incidence was possible using simple functions of the estimated beta-binomial parameters of the distribution of leaf blight and leaf spot. Furthermore, the independence also assures that statistical procedures performed on one of the two diseases alone need not consider the effect of the other disease on observed results.

\section{APPENDIX}

Randomizations. To determine the expected value of the Jaccard index under independence, a randomization procedure was followed. For the $i$ th sampling unit at the leaflet scale, $x_{i} 1 \mathrm{~s}$ and $n-x_{i} 0$ s (in which $n=15$ ) were generated for leaf blight, and these 15 values of $1 \mathrm{~s}$ and $0 \mathrm{~s}$ were randomly assigned to a vector with $n$ elements, $\boldsymbol{x}_{i}$. Next, $y_{i} 2 \mathrm{~s}$ and $n-y_{i} 0$ s were generated for leaf spot, and these values were randomly assigned to a second vector, $\boldsymbol{y}_{i}$. The two vectors were added to produce a third vector, $z_{i}$, representing total disease $\left(z_{i}=x_{i}+y_{i}\right)$ for the $i$ th sampling unit. The number of $3 \mathrm{~s}$, $2 \mathrm{~s}$, and $1 \mathrm{~s}$ in $z_{i}$ were tallied and stored; these values represented $a_{i}$, $b_{i}$, and $c_{i}$, respectively, for the $i$ th sampling unit (specifically, $a_{i, j}$, $b_{i, j}$, and $c_{i, j}$, with the $j$ subscript referring to the values for the $j$ th randomization). This process was repeated for each sampling unit in the data set, and the Jaccard index was then calculated $\left(J_{t, j}\right)$ based on the values of $a_{i, j}, b_{i, j}$, and $c_{i, j}$, as discussed above. This was done $k(j=1,2, \ldots, k)$ times for each data set. $\bar{J}_{t,(\text { ran })}$ was then calculated using

$$
\bar{J}_{t,(\text { ran })}=\sum_{j=1}^{k} J_{t, j} / k
$$


With our data, $k=100$ randomizations were performed for each data set.

At the sampling-unit scale, let $\boldsymbol{X}_{s u}=\left[x_{1, s u}, \ldots, x_{i, s u}, \ldots, x_{N, s u}\right]^{\prime}$ and $\boldsymbol{Y}_{s u}=$ $\left[y_{1, s u}, \ldots, y_{i, s u}, \ldots, y_{N, s u}\right]^{\prime}$ represent vectors of length $N$, in which $x_{i, s u}$ and $y_{i, s u}$ are as defined previously and the prime symbol denotes the matrix transpose operator. Thus, $\boldsymbol{X}_{s u}$ and $\boldsymbol{Y}_{s u}$ represent vectors of $0 \mathrm{~s}$ and $1 \mathrm{~s}$ for an entire data set (one value for each sampling unit, not a vector for a single sampling unit as above [e.g., $\left.\boldsymbol{y}_{i}\right]$ ). The $N$ values of $\boldsymbol{X}_{s u}$ and the $N$ values of $\boldsymbol{Y}_{s u}$ were randomly rearranged separately, and the Jaccard was calculated for the randomized data $\left(J_{s u, j}\right)$. This was done $k=100$ times, and the mean of these Jaccard values $\left(\bar{J}_{s u,(r a n)}\right)$ was determined with equation A1 (with $J_{s u, j}$ for $J_{t, j}$, and $\bar{J}_{s u,(r a n)}$ for $\bar{J}_{t,(\text { ran })}$ ). For this situation, the disease status of the individual sampling units was not preserved, although incidence of both diseases at the field level was fixed.

Extreme values. At the leaflet level, the maximum value of $J$ $\left(J_{t,(\max )}\right)$ was obtained by rearranging the $x_{i} 1 \mathrm{~s}$ and $n-x_{i} 0 \mathrm{~s}$ in $\boldsymbol{x}_{i}$ and the $y_{i} 2 \mathrm{~s}$ and $n-y_{i} 0 \mathrm{~s}$ in $\boldsymbol{y}_{i}$ for each sampling unit $(i=$ $1,2, \ldots, N)$ so that the maximum number of $3 \mathrm{~s}$ (condition in which leaflet has both diseases) was produced in the vector $z_{i}$. For example, if a sampling unit $(n=15)$ had 6 leaflets with blight $\left(x_{i}=6\right)$ and 10 leaflets with spot $\left(y_{i}=10\right)$, then rearranging the leaflets to produce maximum association gives 10 diseased leaflets in total $\left(z_{i}=10\right)$, with $a_{i}=6, b_{i}=0$, and $c_{i}=4$. Note that at least one of the original leaflets in this example must have symptoms of both diseases because $x_{i}+y_{i}>n(6+10>15)$. The minimum value of $J\left(J_{t,(\text { min })}\right)$ was obtained by rearranging the elements of $\boldsymbol{x}_{\boldsymbol{i}}$ and $\boldsymbol{y}_{\boldsymbol{i}}$ so that the minimum number of $3 \mathrm{~s}$ was produced in the vector $z_{i}$. Arranging these same example numbers to obtain minimum association gives 15 diseased leaflets $\left(z_{i}=15\right)$, with $a_{i}=1, b_{i}=5$, and $c_{i}=9$. This procedure was done for each of the $N$ sampling units in a data set; $J_{t,(\max )}$ and $J_{t,(\min )}$ were then calculated. For the maximum and minimum arrangements of each data set, the incidence and heterogeneity of incidence of leaf blight and leaf spot remained the same as the observed; however, incidence and heterogeneity of incidence for total disease changed, and these new values for incidence are denoted as $\hat{p}_{t, z,(\min )}$ and $\hat{p}_{t, z,(\max )}$. Note that maximum association results in the smallest value of total disease incidence; conversely, minimum association results in the largest value of total disease incidence.

At the sampling-unit scale, results were conditioned on the field incidence of both diseases, but not on incidence in the individual sampling units (i.e., it was not possible to preserve the disease status of individual sampling units). To obtain the maximum value of the Jaccard index at the sampling-unit level, the $N$ elements of $\boldsymbol{X}_{s u}$ and $\boldsymbol{Y}_{s u}$ were individually sorted in ascending order and the Jaccard index was calculated for this maximum association of the two diseases $\left(J_{s u,(\max )}\right)$. To obtain the minimum value of $J_{s u}$, the values of $\boldsymbol{X}_{s u}$ were sorted in ascending order and the values of $\boldsymbol{Y}_{s u}$ were sorted in descending order, and the Jaccard index was calculated for this minimum association $\left(J_{s u,(\min )}\right)$. Although $\hat{p}_{s u, x}$ and $\hat{p}_{s u, y}$ (moment estimates of the probability of a sampling unit having blight and spot, respectively) were unchanged with these arrangements, $\hat{p}_{s u, z}$ changed for both minimum and maximum association. Minitab (Minitab Inc.) macros were written to perform all the Jaccard calculations.

\section{ACKNOWLEDGMENTS}

Salaries and research support were provided by state and federal funds appropriated to the Ohio Agricultural Research and Development Center, the Ohio State University.

\section{LITERATURE CITED}

1. Bockus, W. W., Davis, M. A., and Todd, T. C. 1994. Grain yield responses of winter wheat coinoculated with Cephalosporium gramineum and Gaeumannomyces graminis var. tritici. Plant Dis. 78:11-14.

2. Brier, S. E. 1980. Analysis of contingency tables under cluster sampling.
Biometrika 67:591-596.

3. Clifford, P., Richardson, S., and Hémon, D. 1989. Assessing the significance of the correlation between two spatial processes. Biometrics 45: 123-134.

4. Cohen, J. E. 1976. The distribution of the chi-square statistic under cluster sampling from contingency tables. J. Am. Stat. Assoc. 71:665-670.

5. DeGroot, M. H. 1986. Probability and Statistics. 2nd ed. Addison-Wesly Publishing Company, Menlo Park, CA.

6. Dixon, P. M. 1993. The bootstrap and the jackknife: Describing the precision of ecological indices. Pages 290-318 in: Design and Analysis of Ecological Experiments. S. M. Scheiner and J. Gurevitch, eds. Chapman \& Hall, New York.

7. Ellis, M. A. 1995. Integrated Pest Management (IPM). Disease Management Guidelines for Strawberries in Ohio. Ohio State University. Plant Pathology Dept. Series No. 93.

8. Ellis, M. A. 1998. Strawberry Leaf Spot Diseases. Ohio State University. Extension Fact Sheet HYG-3015-95.

9. Fay, R. E. 1985. A jackknifed chi-squared test for complex samples. J. Am. Stat. Assoc. 80:148-157.

10. Ferrandino, F. J. 1998. Past nonrandomness and aggregation to spatial correlation: 2DCORR, a new approach for discrete data. Phytopathology 88:84-91.

11. Firbank, L. G., and Watkinson, A. R. 1985. On the analysis of competition within two-species mixtures of plants. J. Appl. Ecol. 22:503-517.

12. Hughes, G., and Madden, L. V. 1992. Aggregation and incidence of disease. Plant Pathol. 41:657-660.

13. Hughes, G., and Madden, L. V. 1993. Using the beta-binomial distribution to describe aggregated patterns of disease incidence. Phytopathology 83:759-763.

14. Hughes, G., Madden, L. V., and Munkvold, G. P. 1996. Cluster sampling for disease incidence data. Phytopathology 86:132-137.

15. Hughes, G., McRoberts, N., Madden, L. V., and Gottwald, T. R. 1997. Relationships between disease incidence at two levels in a spatial hierarchy. Phytopathology 87:542-550.

16. Legendre, L., and Legendre, P. 1983. Numerical Ecology. Elsevier, New York.

17. Ludwig, J. A., and Reynolds, J. F. 1988. Statistical Ecology. John Wiley $\&$ Sons, New York.

18. Maas, J. L., ed. 1984. Compendium of Strawberry Diseases. The American Phytopathological Society, St. Paul, MN.

19. Madden, L. V. 1989. Dynamic nature of within-field disease and pathogen distributions. Pages 96-126 in: Spatial Components of Plant Disease Epidemics. M. J. Jeger, ed. Prentice-Hall, Englewood Cliffs, NJ.

20. Madden, L. V., and Hughes, G. 1994. BBD—Computer software for fitting the beta-binomial distribution to disease incidence data. Plant Dis. 78:536-540.

21. Madden, L. V., and Hughes, G. 1995. Plant disease incidence: Distributions, heterogeneity, and temporal analysis. Annu. Rev. Phytopathol. 33: 529-564.

22. Madden, L. V., Hughes, G., and Ellis, M. A. 1995. Spatial heterogeneity of the incidence of grape downy mildew. Phytopathology 85:269-275.

23. Madden, L. V., Hughes, G., and Munkvold, G. P. 1996. Plant disease incidence: Inverse sampling, sequential sampling, and confidence intervals when observed mean incidence is zero. Crop Prot. 15:621-632.

24. Madden, L. V., and Nutter, Jr., F. W. 1995. Modeling crop loss at the field scale. Can. J. Plant Pathol. 17:124-137.

25. Manly, B. F. J. 1997. Randomization, Bootstrap and Monte Carlo Methods in Biology. 2nd ed. Chapman \& Hall, London.

26. Martin, M. J., Riedel, R. M., and Rowe, R. C. 1982. Verticillium dahliae and Pratylenchus penetrans: Interactions in the early dying complex of potato in Ohio. Phytopathology 72:640-644.

27. Nelson, S. C., and Campbell, C. L. 1992. Incidence and patterns of association of pathogens in a leaf spot disease complex on white clover in the Piedmont region of North Carolina. Phytopathology 82:1013-1021.

28. Nelson, S. C., and Campbell, C. L. 1993. Disease progress, defoliation, and spatial pattern in a multiple-pathogen disease complex on white clover. Phytopathology 83:419-429.

29. Nelson, S. C., and Campbell, C. L. 1993. Comparative spatial analysis of foliar epidemics on white clover caused by viruses, fungi, and a bacterium. Phytopathology 83:288-301.

30. Newton, M. R., Kinkel, L. L., and Leonard, K. J. 1997. Competition and density-dependent fitness in coexisting foliar pathogens. Ecology 78: 1774-1784.

31. Newton, M. R., Kinkel, L. L., and Leonard, K. J. 1998. Constraints on the use of de Wit models to analyze competitive interactions. Phytopathology 88:873-878.

32. Perry, J. N. 1995. Spatial aspects of animal and plant distribution in patchy farmland habitats. Pages 221-242 in: Ecology and Integrated Farming Systems. D. M. Glen, M. P. Greaves, and H. M. Anderson, eds. 
John Wiley \& Sons, London.

33. Perry, J. N. 1997. Spatial association for counts of two species. Acta Jutlandica 72:149-169.

34. Perry, J. N. 1998. Measures of spatial pattern for counts. Ecology 79: 1008-1017.

35. Perry, J. N. 1998. Measures of spatial pattern and spatial association for insect counts. Pages 21-33 in: Population and Community Ecology for Insect Management and Conservation. J. Baumgartner, P. Brandmayr, and B. F. J. Manly, eds. Balkema, Rotterdam, the Netherlands.

36. Rao, J. N. K., and Scott, A. J. 1981. The analysis of categorical data from complex surveys: Chi-squared tests for goodness of fit and independence in two-way tables. J. Am. Stat. Assoc. 76:221-230.

37. Reynolds, K. M., and Madden, L. V. 1988. Analysis of epidemics using spatio-temporal autocorrelation. Phytopathology 78:240-246.

38. Reynolds, K. M., Madden, L. V., and Ellis, M. A. 1988. Spatio-temporal analysis of epidemic development of leather rot of strawberry. Phytopathology 78:246-252.

39. Snaydon, R. W. 1991. Replacement or additive designs for competition studies? J. Appl. Ecol. 28:930-946.

40. Stitcher, L., Mauch-Manui, B., and Métraux, J. P. 1997. Systemic acquired resistance. Annu. Rev. Phytopathol. 35:235-270.

41. Sun, P., and Zeng, S. 1993. Discriminating synergism and antagonism of spores within species of phytopathogenic fungi. Phytopathology 83:1051-
1054.

42. Turechek, W. W., and Madden, L. V. 1999. Spatial pattern analysis of strawberry leaf blight in perennial production systems. Phytopathology 89:421-433.

43. Turechek, W. W., and Madden, L. V. 1999. Spatial pattern analysis and sequential sampling for the incidence of leaf spot on strawberry in Ohio. Plant Dis. 83:992-1000.

44. van Peer, R., Niemann, G. J., and Schippers, B. 1991. Induced resistance and phytoalexin accumulation in biological control of Fusarium wilt of carnation by Pseudomonas sp. strain WCS417r. Phytopathology 81:728-734.

45. Van Peer, R., Van Kuik, A. J., Rattink, H., and Schippers, B. 1990. Control Fusarium wilt in carnation grown on rockwool by Pseudomonas sp. strain WCS417r and by Fe-EDDHA. Neth. J. Plant Pathol. 96:119-132.

46. Wheeler, T. A., Madden, L. V., Riedel, R. M., and Rowe, R. C. 1994. Distribution and yield-loss relations of Verticillium dahliae, Pratylenchus penetrans, P. scribneri, P. crenatus, and Meloidogyne hapla in commercial potato fields. Phytopathology 84:843-852.

47. Williams, D. A. 1975 . The analysis of binary responses from toxicological experiments involving reproduction and teratogenicity. Biometrics 31:949-952.

48. Xu, X.-M., and Ridout, M. S. 1998. Effects of initial epidemic conditions, sporulation rate, and spore dispersal gradient on the spatio-temporal dynamics of plant disease epidemics. Phytopathology 88:1000-1012. 